\title{
Structure of Receptive Fields in Area 3b of Primary Somatosensory Cortex in the Alert Monkey
}

\author{
James J. DiCarlo, Kenneth O. Johnson, and Steven S. Hsiao \\ Krieger Mind/Brain Institute, Department of Neuroscience, and Department of Biomedical Engineering, Johns Hopkins \\ University, Baltimore, Maryland 21218
}

\begin{abstract}
We investigated the two-dimensional structure of area $3 \mathrm{~b}$ neuronal receptive fields (RFs) in three alert monkeys. Three hundred thirty neurons with RFs on the distal fingerpads were studied with scanned, random dot stimuli. Each neuron was stimulated continuously for $14 \mathrm{~min}$, yielding 20,000 response data points. Excitatory and inhibitory components of each RF were determined with a modified linear regression algorithm. Analyses assessing goodness-of-fit, repeatability, and generality of the RFs were developed. Two hundred forty-seven neurons yielded highly repeatable RF estimates, and most RFs accounted for a large fraction of the explainable response of each neuron. Although the area $3 b$ RF structures appeared to be continuously distributed, certain structural generalities were apparent. Most RFs (94\%) contained a single, central region of excitation and one or more regions of inhibition located on one, two, three, or all four sides of the excitatory center. The shape,
\end{abstract}

area, and strength of excitatory and inhibitory RF regions ranged widely. Half the RFs contained almost evenly balanced excitation and inhibition. The findings indicate that area $3 \mathrm{~b}$ neurons act as local spatiotemporal filters that are maximally excited by the presence of particular stimulus features. We believe that form and texture perception are based on highlevel representations and that area $3 \mathrm{~b}$ is an intermediate stage in the processes leading to these representations. Two possibilities are considered: (1) that these high-level representations are basically somatotopic and that area $3 \mathrm{~b}$ neurons amplify some features and suppress others, or (2) that these representations are highly transformed and that area $3 \mathrm{~b}$ effects a step in the transformation.

Key words: receptive field; somatosensory; cortex; tactile; form; texture; area 3b; SI; monkey; first-order kernel; reverse correlation
Our long-term goal is to elucidate the neural mechanisms of tactile form and texture perception. In this study, we sought to understand the representation of tactile stimuli in area $3 b$ of primary somatosensory (SI) cortex because it is the first cortical area involved in processing the neural signals implicated in form and texture perception. Within area $3 \mathrm{~b}$ regions representing the fingerpad, we described the receptive field (RF) of each neuron as the two-dimensional pattern of excitation and inhibition that determines the neuronal response to complex spatial patterns scanned across the skin.

Several lines of evidence suggest that the neural signals that underlie the perception of tactile form and texture on the glabrous skin of the primate fingerpad are conveyed initially by the population of slowly adapting type I (SAI) mechanoreceptive afferents (for review, see Johnson and Hsiao, 1992). After transmission through the dorsal column nuclei (Mountcastle, 1984) and the thalamus (Poggio and Mountcastle, 1960), these neural signals are projected most densely to area $3 \mathrm{~b}$ of SI cortex (Jones, 1986). Evidence that area $3 b$ is the primary cortical-receiving area for these signals comes from physiological studies showing that, among the SI areas (areas 3a, 3b, 1, and 2), area 3b has the largest cortical surface area devoted to digit representation (Sur

\footnotetext{
Received July 10, 1997; revised Jan. 5, 1998; accepted Jan 8, 1998.

This study was supported by National Institutes of Health Grants NS18787 and NS34086 and by the W. M. Keck Foundation. We thank Drs. S. H. Hendry, V. Mountcastle, G. Poggio, I. A. Twombly, and E. Young for helpful advice and criticism. John Lane, Steve Patterson, and David O'Shaughnessy provided invaluable technical support.

Correspondence should be addressed to Dr. Kenneth O. Johnson, 338 Krieger Hall, Johns Hopkins University, 3400 North Charles Street, Baltimore, MD 21218. Copyright (C) 1998 Society for Neuroscience $0270-6474 / 98 / 182626-20 \$ 05.00 / 0$
}

et al., 1980), the highest proportion of cells responsive to light cutaneous stimulation (Powell and Mountcastle, 1959b; Iwamura et al., 1983; Kaas et al., 1984), cells with the smallest RFs (Paul et al., 1972; Sur et al., 1980, 1985), and the highest proportion of cells responding to static skin indentation (Paul et al., 1972; Sur et al., 1984). Removal of area $3 \mathrm{~b}$ in the monkey markedly reduces the responsiveness of area 1 neurons (Garraghty et al., 1990) and secondary somatosensory (SII) neurons (Pons et al., 1992; see also Pons et al., 1987) to cutaneous stimuli. It also produces profound behavioral deficits in all somatosensory tasks tested, whereas removal of other SI areas produces specific deficits in the tactile discrimination of textures (area 1) and three-dimensional forms (area 2) (Randolph and Semmes, 1974).

Although most area $3 \mathrm{~b}$ neurons appear to have homogeneous, excitatory RFs when probed with punctate stimuli (Mountcastle and Powell, 1959; Sur, 1980), response properties that are more complex than those that would be expected if the RFs were homogeneous have been reported in some area $3 \mathrm{~b}$ neurons. These include excitatory summation (Gardner and Costanzo, 1980a), surround inhibition (Mountcastle and Powell, 1959; Iwamura et al., 1983), directional selectivity (Whitsel et al., 1972; Hyvarinen and Poranen, 1978a; Warren et al., 1986), and orientation selectivity (Pubols and Leroy, 1977; Hyvarinen and Poranen, 1978a; Warren et al., 1986). Likewise, when neurons with RFs on the fingerpads are stimulated with scanned, complex spatial stimuli, almost all yield responses that are more complex than those that can be accounted for by simple, excitatory RFs (Phillips et al., 1988; Bankman et al., 1990; Johnson et al., 1995).

In this study, we scanned surfaces of randomly distributed, raised dots across the RFs of 330 neurons in area $3 \mathrm{~b}$ of three alert 
monkeys. This subjected each neuronal RF to a very large number of different spatial dot patterns. We determined the excitatory and inhibitory structure of each neuron's RF by comparing the instantaneous spike rates with the patterns that evoked them. Most RFs contained a single, well-defined excitatory region and one or more surrounding or offset inhibitory regions. The RFs describe how tactile form on the fingerpad is transformed to a neural representation in area $3 \mathrm{~b}$. This transformation may serve to enhance the presence of spatial features in the stimulus. Alternatively, it may reflect the early stages of a transformational sequence whose end result is a totally different, nonsomatotopic representation that directly underlies form and texture perception.

\section{MATERIALS AND METHODS}

\section{Animals and surgery}

Two male and one female rhesus monkey (Macaca mulatta) weighing 4-5 $\mathrm{kg}$ were used in this study. Each animal was trained to perform a visual detection task during the presentation of tactile stimuli (see below). Although the task was unrelated to the tactile stimuli, it served to maintain the animal in a constant, alert state during recording periods. After the animal was performing the task nearly perfectly, which took a few weeks, surgery was performed to attach a head-holding device and a recording chamber to the skull. Surgical anesthesia was induced with ketamine $\mathrm{HCl}(33 \mathrm{mg} / \mathrm{kg}$, i.m.) and maintained with pentobarbital (10 $\mathrm{mg} \cdot \mathrm{kg}^{-1} \cdot \mathrm{hr}^{-1}$, i.v.). All surgical procedures were done under sterile conditions and in accordance with the rules and regulations of the Johns Hopkins Animal Care and Use Committee and the Society for Neuroscience.

\section{Recording}

Electrophysiological recordings were made in the postcentral gyri of five hemispheres using standard techniques (Phillips et al., 1988; Mountcastle et al., 1991). On each recording day, a multielectrode microdrive (Mountcastle et al., 1991) was loaded with seven quartz-coated platinum/tungsten $(90 / 10)$ electrodes (diameter, $80 \mu \mathrm{m}$; tip diameter, $4 \mu \mathrm{m}$; and impedance, $1-5 \mathrm{M} \Omega$ at $1000 \mathrm{~Hz}$ ). Each electrode was coated with one of three fluorescent dyes (DiI, DiI-C5, or DiO; Molecular Probes, Eugene, OR) to facilitate later histological localization of the recording sites (DiCarlo et al., 1996). The tip of the microdrive was then inserted into the recording chamber, which was filled with physiological saline, and oriented so the electrodes were normal to the skull. The electrodes emerged from the tip of the microdrive separated by $400 \mu \mathrm{m}$ and arranged in a single row. The row of electrodes was oriented normal to the plane of the central sulcus so that three to four electrodes could be positioned in a line across the laminae of area 3b (DiCarlo et al., 1996).

The localization of recordings to area $3 \mathrm{~b}$ was accomplished in two ways: initially, during the experiments, by physiological evidence and later, after euthanasia, by histological methods. During the experiments, we relied on the characteristic progression of RF locations as each electrode was advanced through area 1 into area $3 \mathrm{~b}$ (Merzenich et al., 1978; Sur et al., 1984). This progression was nearly identical in all five hemispheres. At the first sign of neural activity, the cutaneous RFs usually occupied most, or a portion, of a single digit and could be found on either the glabrous or hairy skin. As the electrode was advanced, the location of the RF shifted toward the base of the finger and often spread to the palmer whorls (at $\sim 1500-2000 \mu \mathrm{m}$ below the first sign of neural activity). As the electrode was advanced further, the RF location began to move back onto the glabrous surface of the proximal phalanx and was much more localized. Later histological analysis showed that this reversal corresponded closely to the border between areas 1 and $3 \mathrm{~b}$. Over the next 500-1000 $\mu \mathrm{m}$, the RF locations continued to move distally along the glabrous surface of the digit without jumps or discontinuities. Neurons with RFs located on one of the distal fingerpads in area $3 \mathrm{~b}$ were typically located in a region $2000-3000 \mu \mathrm{m}$ below the first signs of neural activity. All neurons that met the following criteria were studied using the stimulus procedures described below: (1) the neuron's action potentials were well isolated from the noise, (2) the neural RF was located on one of the distal fingerpads (digits 2-5), and (3) the stimulus drum and the hand (see below) could be positioned so that the RF was centered on the portion of the fingerpad in contact with the stimulus. Using manual probes, we encountered very few neurons $(<5 \%)$ with RFs that extended over multiple digits, but we cannot eliminate the possibility that multidigit RF components might be revealed with quantitative RF-mapping techniques spanning more than a single fingerpad.

On each successive day of recording, we shifted the position of the line of microelectrodes $\sim 300 \mu \mathrm{m}$ medial (or lateral) from the position of the previous day. Thus, after $\sim 15-20$ recording days in each hemisphere, we sampled from the entire cortical volume devoted to the glabrous distal digit representation (digits 2-5) in area $3 \mathrm{~b}$.

Action potentials from each electrode were discriminated from the background noise with an amplitude discriminator. A continuous record of the stimulus location and the times of occurrences of action potentials, stimulus events, and behavioral events were stored in a computer with an accuracy of $0.1 \mathrm{msec}$. At the end of each day, the electrodes were withdrawn, two drops of dexamethasone phosphate $(0.1 \%)$ and gentamycin $(0.002 \%)$ were applied to the dura, Gelfoam (Upjohn, Kalamazoo, MI) soaked in sterile saline was inserted into the recording chamber, and the chamber was sealed.

\section{Stimuli}

The stimuli were patterns of raised dots (see Figs. 1,2) or letters fabricated from sheets of photosensitive plastic that are water-soluble until exposed to UV light (Toyoba Printight plastics, EF series). A raised stimulus pattern was produced by laying a photographic negative of the pattern over the plastic sheet and exposing it to UV light, which polymerizes and hardens the exposed plastic. The portion of the surface layer not exposed to UV light is scrubbed off lightly in water, leaving a raised pattern whose thickness equals the thickness of the original water-soluble layer ( 400 or $500 \mu \mathrm{m}$; see below). The etching precision is limited only by the precision of the photographic negative. The stimulus pattern was specified in Postscript and printed at 3386 dots per inch. After construction, sheets containing the stimulus patterns were wrapped around and glued to cylindrical drums, $320 \mathrm{~mm}$ in circumference, which were mounted on a rotating drum stimulator (Johnson and Phillips, 1988) (see Fig. 1).

The same photographic negative with a single random dot pattern, 28 $\mathrm{mm}$ wide and $250 \mathrm{~mm}$ long, was used to construct the random dot stimuli for all three monkeys. In the experiments performed on one monkey, the pattern was trimmed to $175 \mathrm{~mm}$ long to make room for a segment with oriented bars. Otherwise, the stimuli were identical in the three monkeys. Each dot was $400 \mu \mathrm{m}$ high (in relief) and $500 \mu \mathrm{m}$ in diameter at its top, with sides that sloped away at $60^{\circ}$ relative to the surface of the drum. The location of the center of each dot was determined by selecting two random numbers from a uniform random number generator and scaling them to the height $(28 \mathrm{~mm})$ and width $(250 \mathrm{~mm})$ of the pattern. The dot centers were specified to a precision of $1 \mu \mathrm{m}$, and dots were allowed to overlap when the random number generator placed them closer than the dot diameter. Dots were added until the average dot density equaled 10 dots per square centimeter (i.e., 700 dots on a surface $28 \mathrm{~mm}$ wide $\times 250$ $\mathrm{mm}$ long). Random dot patterns are unbiased in the sense that all possible patterns with the specified dot density are equally likely and the probability of a repeated pattern is virtually zero. The dot density (10 dots $/ \mathrm{cm}^{2}$ ) was chosen to satisfy two constraints. First, it was large enough that, at each position on the pattern, the RF was often stimulated by several dots. Such stimulation enables the detection of inhibitory RF regions as a decrement in the response to a simultaneously stimulated excitatory RF region (see the Discussion). Second, preliminary studies showed that higher dot densities resulted in lower average firing rates in primary afferent SAI neurons, an important source of input to area $3 \mathrm{~b}$. A second stimulus pattern consisted of embossed Helvetica letters $8 \mathrm{~mm}$ high that were composed of lines $500 \mu \mathrm{m}$ wide and $500 \mu \mathrm{m}$ in relief (Phillips et al., 1988).

After one or more neurons with overlapping RF locations were isolated with one or more of the electrodes, a drum with one of the stimulus patterns was positioned over the fingerpad so that all of the neural RFs were located in the cutaneous region contacting the drum surface. During stimulus presentation, the orientation and the angular velocity of the drum were adjusted to produce proximal-to-distal stimulus movement at $40 \mathrm{~mm} / \mathrm{sec}$ across the skin surface. Contact force was controlled by a torque motor set to deliver $30 \mathrm{gm}$ of force to the fingerpad. The drum was positioned initially so that the cutaneous contact region was entirely within the random dot pattern and the center of the contact region was $\sim 5 \mathrm{~mm}$ from the edge of the long side of the pattern. After each revolution, the drum was stepped $200 \mu \mathrm{m}$ along its axis of rotation (Fig. 1). For most of the neurons presented here, the drum completed 100 revolutions (i.e., sweeps) and thus stepped a total distance of $20 \mathrm{~mm}$, 


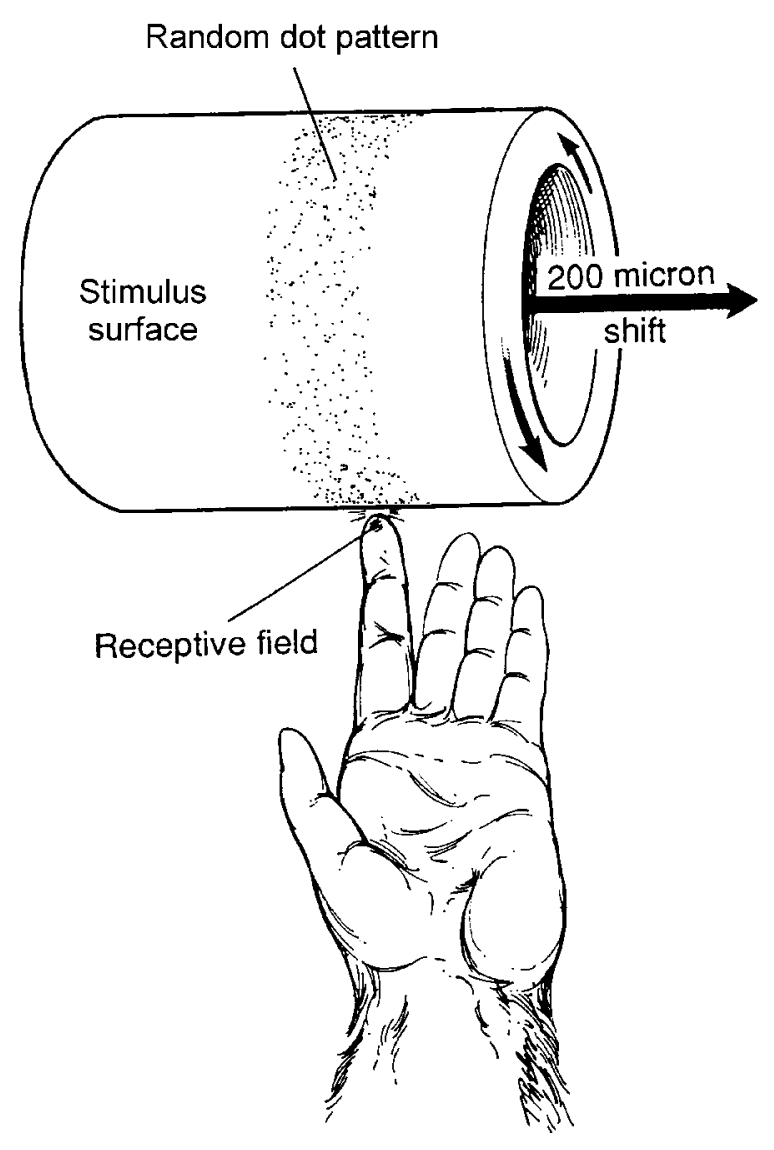

Figure 1. Stimulus. The stimulus pattern was a field $(28 \times 250 \mathrm{~mm})$ of randomly distributed, raised dots (see Fig. 2) mounted on the surface of a drum (Johnson and Phillips, 1988). The dots were $400 \mu \mathrm{m}$ in relief and $500 \mu \mathrm{m}$ in diameter and averaged 10 per $\mathrm{cm}^{2}$. The drum was lowered onto the skin of the distal fingerpad containing the neural RF with a controlled force of $30 \mathrm{gm}$. The hand and finger were held fixed from below (data not shown). The drum rotated at a constant angular velocity to produce proximal-to-distal motion at $40 \mathrm{~mm} / \mathrm{sec}$. After each rotation, the drum was translated by $200 \mu \mathrm{m}$ along its axis of rotation. The recording period yielding the data for a single RF estimate typically involved 100 revolutions and lasted 14 min (see Materials and Methods for details).

which took $\sim 14$ min. Two hundred marker impulses triggered at fixed, equal increments around the drum hub were used to determine the position of the stimulus relative to the occurrence of each action potential with an accuracy of $0.1 \mathrm{msec}$ (Johnson and Phillips, 1988).

\section{Histology}

After several weeks of recording from each hemisphere, the animal was deeply anesthetized with sodium pentobarbital $(65 \mathrm{mg} / \mathrm{kg}$, i.v. $)$ and perfused transcardially with phosphate buffer followed by cold, phosphate-buffered $3 \%$ paraformaldehyde, $\mathrm{pH}=7.4$. Frozen tissue sections $(50 \mu \mathrm{m})$ were mounted but were not stained. Electrode tracks were visualized using fluorescent dye traces left by each electrode (DiCarlo et al., 1996). The electrode tracks and borders of each section were traced into a computer data file (using Neurolucida). These data files were used to construct a three-dimensional rendering of the electrode tracings (using AutoCAD). Neuron locations were then determined along these tracings using microdrive depth measurements. The tissue sections were then stained with cresyl violet, and the area $3 \mathrm{~b}$ borders were defined according to the criteria of Powell and Mountcastle (1959a). For this study, we simply confirmed that all neurons included in the final sample were located in area $3 \mathrm{~b}$. Detailed laminar analysis of these results will be discussed in a future paper.

\section{RF estimation}

The goal of the analysis presented here was to use the pattern and strength of firing to infer the two-dimensional pattern of RF excitation and inhibition on the skin. We assumed that each small region of skin had a positive, negative, or zero effect on the firing rate when stimulated and that the instantaneous firing rate was equal to the sum of these effects. More specifically, we subdivided a $10 \mathrm{~mm}$ square region of skin containing the RF into a grid of $625(25 \times 25)$ subregions, each $400 \mu \mathrm{m}$ square. The method used to estimate the contribution of each subregion is applicable to a wide range of stimulus patterns. However, because our stimulus pattern is random, the estimation method has a simple explanation. Each of the subregions was stimulated by a passing dot $\sim 1000$ times (each region of skin was stimulated by $50 \mathrm{~cm}^{2}$ of the stimulus pattern, which contained $\sim 500$ dots, and each dot passed over each $400 \times$ $400 \mu \mathrm{m}$ subregion twice). On each of those occasions, the dot passed over the subregion for $10 \mathrm{msec}(400 \mu \mathrm{m}$ at $40 \mathrm{~mm} / \mathrm{sec})$; thus, each subregion was stimulated for a total of $10 \mathrm{sec}$ out of the total recording time of 14 min. The average firing rate during the 1000 instances when a subregion was stimulated is the overall mean firing rate (because all other sites were stimulated randomly) plus the effect of the specific region under consideration. For example, if the subregion was strongly inhibitory, then the mean firing rate during stimulation of that subregion was much lower than the overall mean firing rate. If the subregion was only mildly inhibitory, the mean firing rate was only slightly less than the overall mean. In other words, the deviation (positive or negative) from the overall mean firing rate was graded and proportional to the strength of the effect (excitatory or inhibitory). The RF maps presented in this paper are, in effect, maps of the mean deviations in firing rate produced by each subregion in the RF. The method just described is related closely to reverse correlation (de Boer and Kuyper, 1968; Jones and Palmer, 1987). The method we actually used was a modified form of linear regression, which includes reverse correlation as a special case when the stimulus pattern is perfectly random. Linear regression is more general because it provides a least-squared-error solution whether the patterns are random or not. The randomness of the pattern simply makes the estimates more robust (less susceptible to noise in the response). The details follow.

Aligning the response with the stimulus. Each neuron responded to the random dot pattern with a stream of up to 60,000 action potentials recorded continuously for the $14 \mathrm{~min}$ or so required to present the entire random dot pattern. To bring the neural response into alignment with the stimulus, we assigned each action potential a $(x, y)$ position on the surface of the drum corresponding to the location of the stimulus pattern at the time of the action potential. The $x$ location (distance in the scanning direction from the beginning of the random dot pattern) was determined by a digital shaft encoder. The $y$ location was determined by the axial position of the drum. The precision of the method is better than $8 \mu \mathrm{m}$ (Johnson and Phillips, 1988). The resulting spatial raster (see, e.g., Fig. $2 D$ ) is referred to as a spatial event plot (SEP).

This spatial raster of impulse locations was converted to a twodimensional histogram by dividing the space into a rectangular grid of $400 \times 400 \mu \mathrm{m}$ bins and counting the number of impulses in each bin. Four hundred micrometers were selected as the unit of spatial resolution because this distance is less than half the mean spacing of SAI and rapidly adapting (RA) primary afferents innervating the fingerpad (Darian-Smith and Kenins, 1980) and is thus able to approximate the spatial details of the afferent inputs even when they are spaced irregularly. Along the scanning axis of the stimulus (proximal-distal finger axis), $400 \mu \mathrm{m}$ is equivalent to $10 \mathrm{msec}$ ( $40 \mathrm{~mm} / \mathrm{sec}$ scanning velocity). Along the axis orthogonal to the scanning direction, $400 \mu \mathrm{m}$ captures data from two sweeps because each sweep is displaced $200 \mu \mathrm{m}$ from the previous one (see above). A typical stimulus run comprised 100 sweeps of the stimulus, which yielded a response histogram with 20,000 bins.

A comparable histogram describing the random dot stimulus (stimulus histogram) was created in two ways. In the simpler approach, each $400 \times$ $400 \mu \mathrm{m}$ bin containing one or more dot centers was assigned the value 0.4 ( $\mathrm{mm}$ in relief), and all other bins were filled with zeros. In the more complicated approach, each histogram bin was assigned a number proportional to the raised dot area within its boundaries. The results were unaffected by the choice of method, so the simpler approach was used.

To determine the stimulus interactions that produced the neural response, we aligned the stimulus and the response histograms. Even though position signals from the drum stimulator were accurate to the nearest $8 \mu \mathrm{m}$ (Johnson and Phillips, 1988) and spike times were recorded with an accuracy of $0.1 \mathrm{msec}$, lack of knowledge of the exact spatial relationship between the stimulus drum and the RF as well as an unknown delay between the stimulus and the cortical action potentials made the initial alignment of the stimulus and the response histograms 
approximate. However, exact alignment is not critical. If the stimulus is shifted relative to the response, the whole RF excitatory and inhibitory structure is simply shifted within the $10 \times 10 \mathrm{~mm}$ RF grid. Nevertheless, we standardized the alignment by shifting the response histogram to produce the maximum absolute cross-correlation (usually positive but occasionally negative) between the stimulus and the response histograms. Because of this, the RF peak value (usually excitatory) was centered in the RF grid.

Estimating the RF map. We assumed that the impulse rate in each response bin $[r(n) ; n=$ current response bin] was the summed effect of several factors that included a possible constant background discharge rate ( $b_{0}$ in the equation below), the summed excitatory and inhibitory effects of all of the stimulus elements within the RF, possible nonlinear interactions between the stimulus elements $\left(r_{\mathrm{nl}}\right)$, and a random component $(\epsilon)$ :

$r(n)=b_{0}+b_{1} x_{1}(n)+b_{2} x_{2}(n)+\ldots+b_{625} x_{625}(n)+r_{\mathrm{nl}}(n)+\varepsilon$

(when sum of all effects $>0$ ),

$=0$

$$
\text { (when sum of all effects }<0 \text { ). }
$$

The product $b_{i} x_{i}(n)$ represents the contribution of the stimulus $x_{i}(n)$ in the $i$ th RF bin at time step $n$. The stimulus $x_{i}$ in the $i$ th bin was assigned a value equal to the height of the stimulus in the bin at that moment $(0.4$ $\mathrm{mm}$ if a dot was present; 0 if it was not). The coefficients $b_{1}$ to $b_{625}$ represent the weights (positive, excitatory; negative, inhibitory) given to the stimuli $x_{1}$ to $x_{625}$ in the $25 \times 25$ subregions of the RF. The weight matrix specified by $b_{1}$ to $b_{625}$ specifies the pattern of excitation and inhibition within the RF; for brevity, it will be referred to as the RF.

This formulation resulted in one equation for each bin (i.e., time step) in the response histogram except for those near the beginning and end of each sweep where the random dot pattern did not cover the RF completely. The actual number of equations depended on the number of sweeps and the alignment of the stimulus and response but was typically $\sim 20,000$ equations.

The unknown weights that specify the RF $\left(b_{1} \ldots, b_{625}\right)$ can be estimated in a variety of ways, including neural net (Johnson et al., 1995), kernel (Marmarelis and Marmarelis, 1978), and regression (Draper and Smith, 1981) methods. These methods all yield essentially the same result, the set of weights that minimizes the mean-squared difference between the predicted and observed responses. We accomplished this minimization by adhering as closely as possible to the standard methods of multiple linear regression (Draper and Smith, 1981). The main complication relates to the nonlinearity inherent in an inability of a neuron to produce negative impulse rates (threshold effect). The details are presented in Appendix. The nonlinear response component $r_{\mathrm{nl}}(n)$ is, by definition, the repeatable (nonrandom) part of the response that cannot be explained by the linear RF. The magnitude of this response component is estimated by averaging repeated sweeps (see Appendix).

After the RF is estimated, issues related to the quality of the estimate arise. We address these under the headings of repeatability, goodnessof-fit, and generality. The methods used to address the questions of repeatability and generality are presented in the Results. The question of goodness-of-fit is what fraction of the explainable variation in the data (i.e., the repeatable variation) is actually explained by the linear interactions described by the RF $\left(b_{0} \ldots, b_{625}\right)$. The computation of goodnessof-fit is presented in Appendix.

\section{RESULTS}

Random dot patterns were scanned across the RFs of 330 neurons in area $3 \mathrm{~b}$ of five hemispheres in three alert monkeys. A neuron with an RF located on one of the distal fingerpads was excluded from the study only if the drum stimulator could not be positioned to bring the RF, mapped with a manual probe, well within the contact region between the skin and the stimulus surface. Even neurons that were marginally responsive to manual probing were studied with the idea that the random dot pattern might uncover responsiveness that was not evident with simpler probing. Of these 330 neurons, 298 (90\%) were sufficiently modulated by the random dot pattern so that their responses could be confidently aligned with the stimulus pattern and their RFs determined (see
Materials and Methods). The mean firing rate evoked by the random dot patterns was 31.9 impulses/sec.

We first present a description of the RF structures observed in area $3 \mathrm{~b}$ followed by several key measures of those RFs, such as RF areas, magnitudes, orientations, and aspect ratios. Then, we address issues related to the quality of these RF estimates under the headings of repeatability, goodness-of-fit, and generality.

\section{A typical RF}

An example of a typical area $3 \mathrm{~b}$ RF is shown in Figure 2, which illustrates the conventions adhered to throughout this paper. The square, gray scale image illustrated in Figure $2 A$ shows the excitatory (dark) and inhibitory (light) weights that best describe the response patterns illustrated in Figure 2, $D$ and $E$. The RF weights are displayed as though they were viewed through the back of the finger with the tip of the finger pointing to the left of the figure. The stimulus pattern (Fig. $2 B$ ) is moving from right to left beneath the fingerpad at $40 \mathrm{~mm} / \mathrm{sec}$. The relative motion is that that would occur if the RF (Fig. $2 A$ ) was scanned from left to right across the stimulus pattern (Fig. $2 B$ ).

The gray scale RF plot illustrated in Figure $2 A$ is a $25 \times 25$ grid of weights, each representing the influence of a stimulus dot within a single $400 \times 400 \mu \mathrm{m}$ patch of skin. Each RF weight value can be interpreted as the instantaneous change in firing rate that occurs when its $400 \times 400 \mu \mathrm{m}$ skin region is depressed by a stimulus feature $400 \mu \mathrm{m}$ high (the dot relief) moving proximally to distally at $40 \mathrm{~mm} / \mathrm{sec}$. For purposes of visualization, each RF was normalized by dividing all weights by the absolute value of the largest weight, thereby restricting the values to the range from -1 to +1 . The "background" gray level (the gray level around the border, which is $50 \%$ black) represents the region where dots had no average effect (zero RF weight), with darker regions representing excitatory effects and lighter regions representing inhibitory effects. Black regions ( $100 \%$ black) correspond to normalized $\mathrm{RF}$ values of +1 . White regions $(0 \%$ black) correspond to a normalized RF value of -1 and occur only when the absolute maximum RF value is inhibitory, which happened infrequently.

The RF map in Figure $2 A$ shows that this neuron had a region of intense excitation followed by a slightly larger region of inhibition and that both regions were oriented at $\sim 45^{\circ}$ relative to the proximal-distal axis of the finger. The weights and their spatial organization were determined by multiple regression of the neural response on the stimulus as described in Materials and Methods and Appendix. The relationship between the weight pattern and the responses can be seen by inspecting Figure $2 B-D$. Whenever one or more dots occurred anywhere within the darkened region of the RF, the probability of firing increased. Occurrence of dots only within the white, inhibitory region of the RF had no effect because this neuron, like most neurons in the study, had no background discharge to be inhibited. However, whenever one or more dots occurred in the inhibitory region at the same time that dots occurred in the excitatory region, the probability of firing was reduced relative to that expected from stimulation of the excitatory region alone. Three instances are shown in Figure $2 B-D$ (the RF weight pattern in Figure $2 B$ is lightened so the stimulus dots within the excitatory region can be seen). The left example shows an instant in the ongoing interaction between the RF and the stimulus pattern in which three dots happen to lie within the excitatory region of the RF. This alignment predicts an intense response, which is displayed at the tip of the left vertical arrow in Figure $2 C$. The actual response is displayed at the comparable location in Figure 2D. The second (middle) example illustrates an 


\section{A. Linear receptive field (RF)}
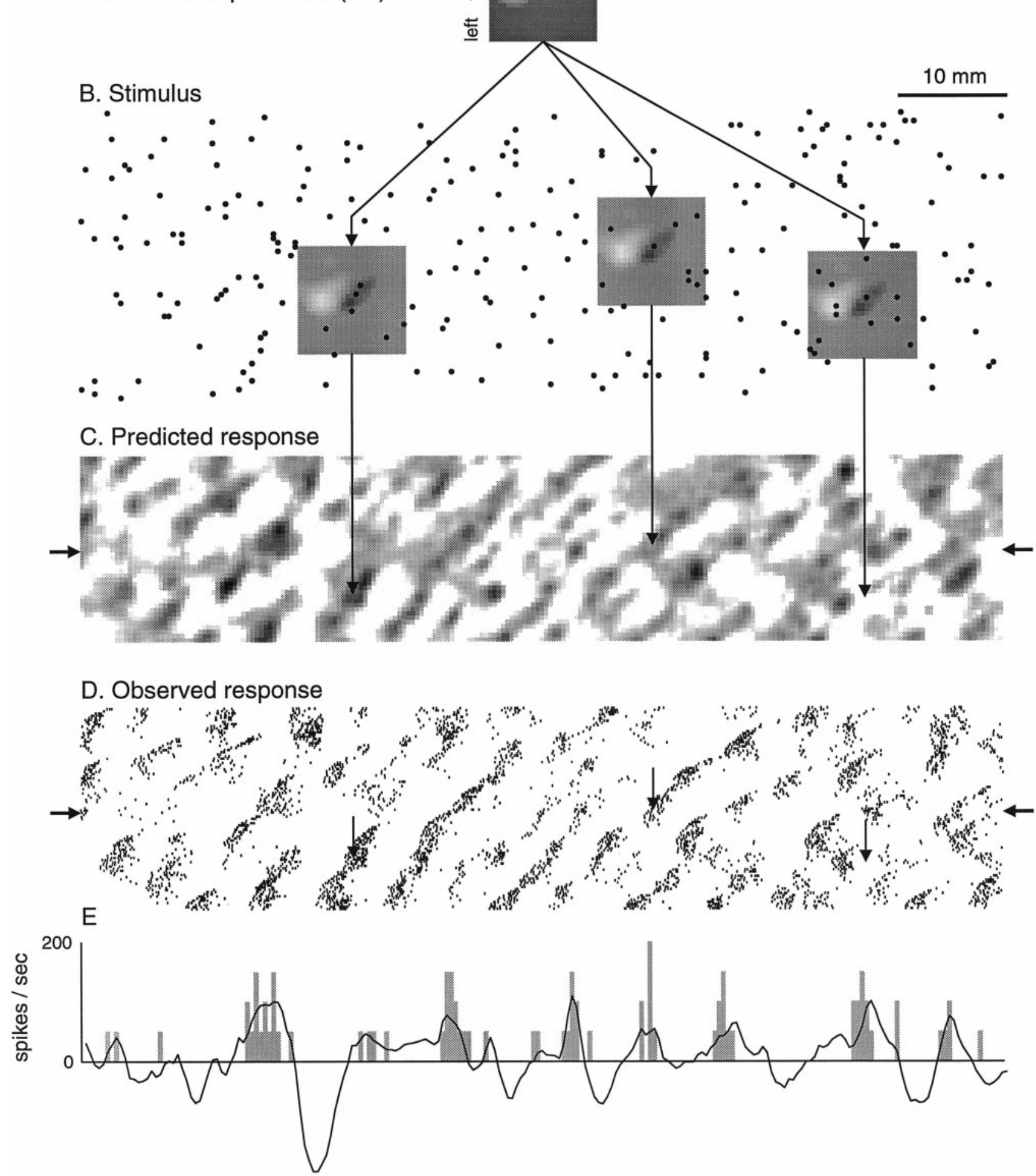

Figure 2. A typical neural response and the resulting RF estimate. $A$, RF estimate. The gray scale represents the grid of weights $(25 \times 25$ bins $=10 \times$ $10 \mathrm{~mm}$ ) that best described the response of the neuron to the random dot stimulus pattern (see Materials and Methods). The RF diagram is meant to represent excitatory and inhibitory skin regions viewed through the back of the finger as the finger points to the left and the stimulus pattern moves from right to left under the finger. The background gray level (50\% black) represents the region where dots had no (linear) effect on the neural response, with darker levels representing excitatory regions where dots increased the probability of firing and lighter levels representing regions where dots decreased the probability of firing. $B$, A portion of the random dot stimulus pattern with the RF superimposed at three locations. The intensity of the RF gray scale has been reduced so the stimulus dots can be seen. $C$, Neural impulse rates predicted by convolving the RF $(A)$ with the (Figure legend continues) 

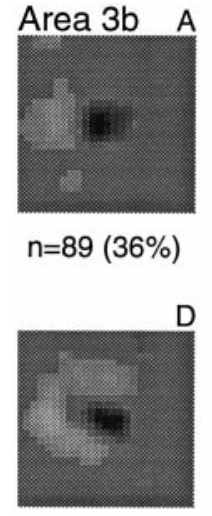

$n=35(14 \%)$

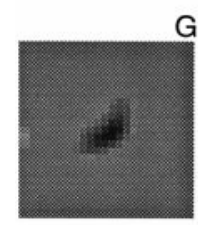

$n=2(1 \%)$

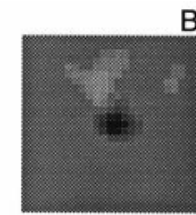

$\mathrm{n}=39(16 \%)$

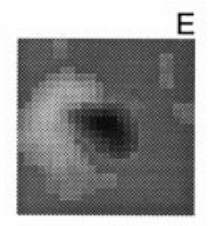

$\mathrm{n}=22(9 \%)$

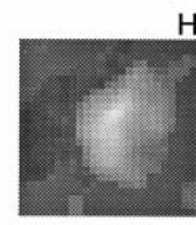

$\mathrm{n}=2(1 \%)$

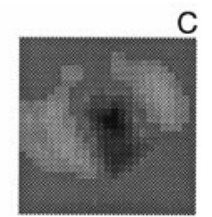

$\mathrm{n}=38(15 \%)$

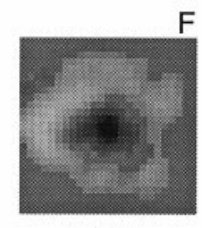

$n=8(3 \%)$

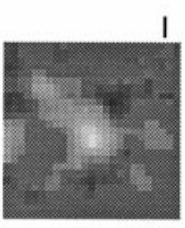

$n=12(5 \%)$
Figure 3. RF structures observed in area 3b. Each panel gives a typical example of the type, the total number of RFs fitting the description, and their percent of the total RF sample $(n=247)$. The types are shown in decreasing order of frequency. $A$, A single inhibitory region located on the trailing (distal) side of the excitatory region. $B$, A region of inhibition located on one of the three nontrailing sides of the excitatory region. $C$, Two regions of inhibition on opposite sides of the excitatory region. $D$, Inhibition on three sides of the excitatory region. $E$, Inhibition on two contiguous sides of the excitatory region. $F$, A complete inhibitory surround. $G$, An excitatory region only. $H$, RF dominated by inhibition. $I$, RFs not easily assigned to one of the preceding categories.

alignment in which only a single dot lies within the excitatory region. The predicted (Fig. 2C) and actual (Fig. 2D) responses are much less intense than are those in the first example. The third (right) example shows an alignment with a stimulus dot at exactly the same place within the excitatory subfield but also with two dots within the inhibitory subfield. The predicted (Fig. 2C) and actual (Fig. 2D) result is a cessation of firing. A typical experiment produced $\sim 20,000$ stimulus-response combinations of the kind illustrated in these three examples, which provided the basis for the precise estimation of the RF weights. Figure $2 E$ shows a continuous trace of the predicted and observed firing rates across the single scan indicated by the arrows to the left and right of Figure 2, $C$ and $D$.

The RF illustrated in Figure 2, like $36 \%$ of the neurons in this study (see Fig. 3), has a single region of inhibition displaced distally relative to the center of excitation. Because the random dot patterns were scanned across the RF of the neuron at one velocity and in the proximal-to-distal direction, space and time are confounded in that direction. For example, the inhibitory region in the RF illustrated in Figure 2, whose center is displaced
$2.4 \mathrm{~mm}$ distal to the center of excitation, could have resulted from inhibition displaced distally from the center of excitation by 2.4 $\mathrm{mm}$, from inhibition (or suppression) delayed by $60 \mathrm{msec}$ relative to the excitation $(2.4 \mathrm{~mm}$ at $40 \mathrm{~mm} / \mathrm{sec})$, or from some combination of the two possibilities.

\section{Characterization of the RFs}

Because some neurons were not well driven by the random dot pattern, the RFs derived from such neurons were flat, variable, and not suitable for quantitative analysis. To identify those neurons, we adopted a criterion based on estimates of the noise and signal in the estimated RF (see below). Using this criterion, we removed 51 of the 298 neurons (15\%), reducing the sample on which more extensive analyses were done to 247 neurons.

Although the RF structures ranged widely, as can be seen by examining the many examples presented in this paper, they typically consisted of a central region of excitation combined with surrounding, flanking, or offset regions of inhibition. To give a general picture of the RFs, we assigned each RF to one of the nine types illustrated in Figure 3. Thirty-six percent ( 89 of 247) of the RFs had a single inhibitory region located on the trailing side of the excitation (i.e., along the direction in which the pattern was scanned); $16 \%$ (39 of 247) had a single region of inhibition that was located on one of the three nontrailing sides of the excitatory region; $15 \%$ (38 of 247) had two regions of inhibition on opposite sides of the excitation; $14 \%$ (35 of 247) had inhibition on three sides of the excitatory region; $9 \%$ (22 of 247) had inhibition on two contiguous sides of the excitatory region; $3 \%$ (8 of 247) were clear examples of an excitatory center with an inhibitory surround; $1 \%$ (2 of 247) had only an excitatory region; and $1 \%$ (2 of 247) were dominated by inhibition. Only 5\% (12 of 247) could not be assigned to any of these categories. Although we have grouped the RFs to illustrate the proportions of RF types found in area $3 \mathrm{~b}$, it should be emphasized that the RF examples shown in Figure 3 do not represent distinct, separate groups; rather, they are samples from an apparently continuous distribution of RF structures.

Neuronal responses are affected not only by the relative positions of the excitatory and inhibitory regions in the RF but also by their areas, amplitudes, shapes, and orientations. In the following sections, we describe these and other properties. In each graph, we chose RFs to illustrate the points in that graph and also to illustrate the distribution of RFs we encountered in area $3 \mathrm{~b}$. Overall, a sixth of the total sample (43 of 247 RFs) is illustrated.

\section{RF area}

Convergence within the nervous system results in a loss of specificity for location and, it is presumed, a gain in specificity of some other kind. The area of each RF that is sensitive to dot stimulation provides an index of this convergence and therefore an index of the degree to which the neural representation of tactile stimuli has changed.

Excitatory (inhibitory) RF area was computed as the number of positive (negative) RF bins exceeding a threshold multiplied by the area of skin represented by each bin $(400 \mu \mathrm{m} \times 400 \mu \mathrm{m}=$

\section{$\leftarrow$}

random dot stimulus $(B)$ and by clipping negative values to zero. Darker regions correspond to higher predicted rates. The arrows extending from $B$ to $C$ point to the predicted impulse rates for each of the three RF positions in $B$. $D$, Observed response of this neuron. Each tick mark indicates the occurrence of a single spike. The plotted position of each spike was determined by the location of the stimulus pattern at the instant the spike occurred (SEP). The three vertical arrows indicate the responses at the stimulus locations corresponding to the three predicted responses in $C$. E, Predicted (black line) and observed (gray histogram) impulse rates in a single scan are indicated by the arrows at the sides of $C$ and $D$. Predicted rates $<0$ correspond to periods in which the summed inhibitory effects exceed the summed excitatory effects. 

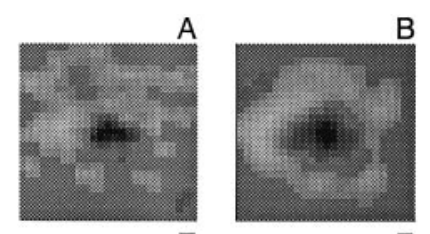

$\mathrm{E}$
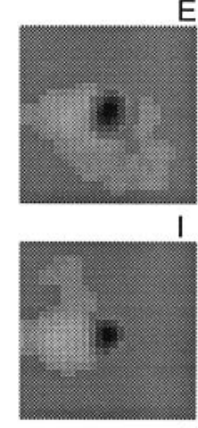

M
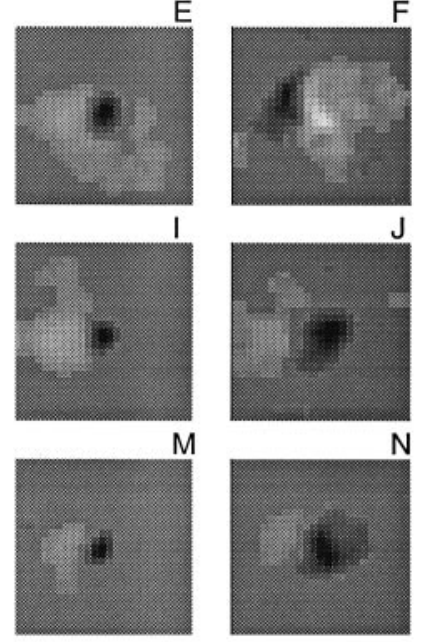

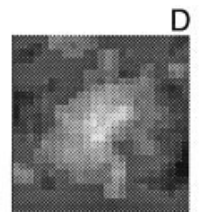

G
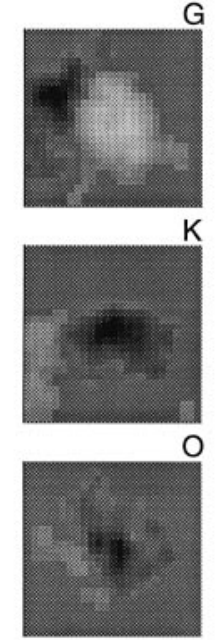
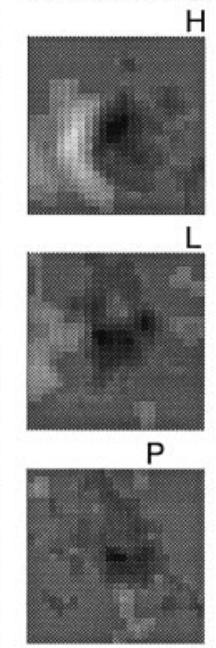

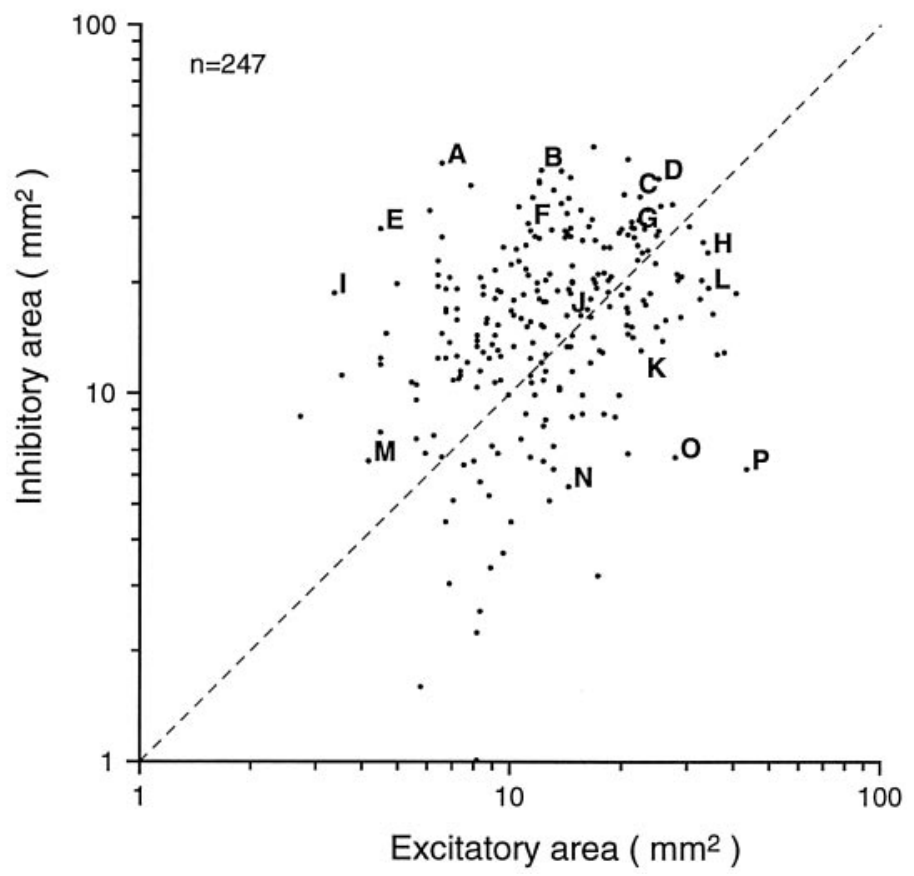

Figure 4. Scatter plot of excitatory and inhibitory RF areas. Excitatory area was measured as the total positive area in the thresholded RF (positive RF regions with values $>10 \%$ of the peak absolute RF value, see Results). Inhibitory area was measured as the total negative-thresholded RF area (negative $\mathrm{RF}$ regions with absolute values $>10 \%$ of the peak absolute RF value). The sample RFs on the left $(A-P)$ are meant to illustrate a wide range of combinations of excitatory and inhibitory areas. The letter above each RF is keyed to a point in the scatter plot (right).
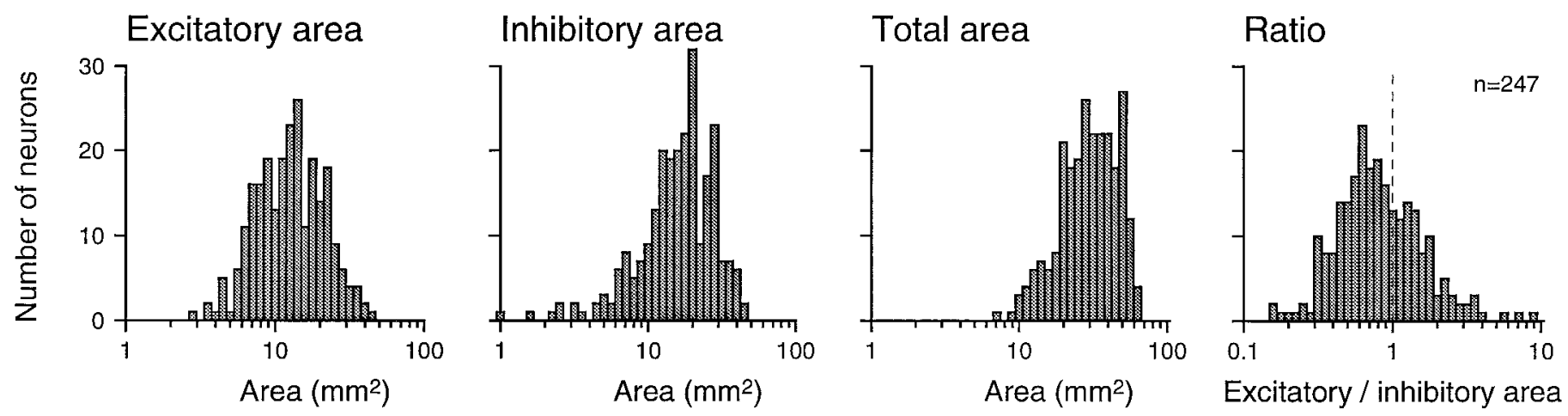

Figure 5. Distributions of RF areas. The $y$-axis values represent numbers of neurons ( $n=247$ neurons in all graphs). From left to right, the $x$-axis values represent excitatory RF area, inhibitory RF area, total RF area (sum of excitatory and inhibitory areas), and the ratio of excitatory to inhibitory area, all on logarithmic scales.

$0.16 \mathrm{~mm}^{2}$ ). If the RF excitatory and inhibitory values dropped off steeply at their boundaries and there was no noise, no special considerations would be required to estimate the RF excitatory and inhibitory areas. However, that is not the case. The RF estimates do contain noise (see below), and their excitatory and inhibitory profiles often decline gradually near their borders (see Fig. 6), making the exact location of their boundaries a matter of definition. The definition used in this study is described in Appendix. Briefly, each RF was first smoothed with a Gaussian filter $(\mathrm{SD}=300 \mu \mathrm{m})$ and then thresholded at a value equal to $10 \%$ of the absolute maximum RF value, which was usually the peak excitatory value. A final stage eliminated small isolated, noiseinduced islands of positive and negative pixels. All RF parameters (except RF noise, discussed below) were computed after these filtering and thresholding steps.
Distributions of excitatory area, inhibitory area, excitatory to inhibitory area ratio, and total RF area are shown in Figures 4 and 5. Excitatory and inhibitory areas were distributed without any obvious clustering and without strong correlation (Pearson correlation $=0.262$ ). Both excitatory and inhibitory areas varied widely. The minimum, mean, and maximum excitatory areas were 3,14 , and $43 \mathrm{~mm}^{2}$, respectively. The comparable inhibitory areas were 1,18 , and $47 \mathrm{~mm}^{2}$, respectively. When plotted in logarithmic coordinates, neither the excitatory nor the inhibitory distribution was significantly different from a normal distribution $(p>0.05$; Kolmogorov-Smirnoff one-sample test; SPSS). The bivariate (excitatory and inhibitory area) $\log$ plot shown in Figure 4 has means of 1.100 and $1.189 \log _{10}$ units), SDs of 0.224 and $0.262 \log _{10}$ units, and a correlation of 0.327 . The geometric mean inhibitory area was $23 \%$ larger than was the geometric mean excitatory area, and 

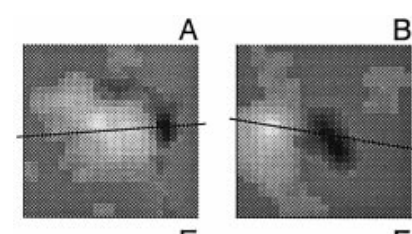

$\mathrm{E}$
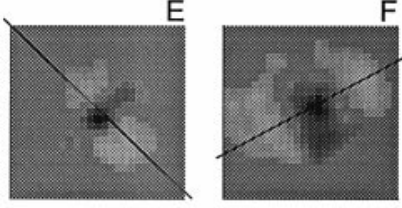

$\mathrm{F}$
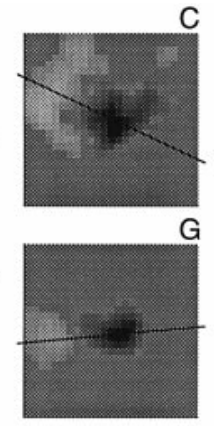

G
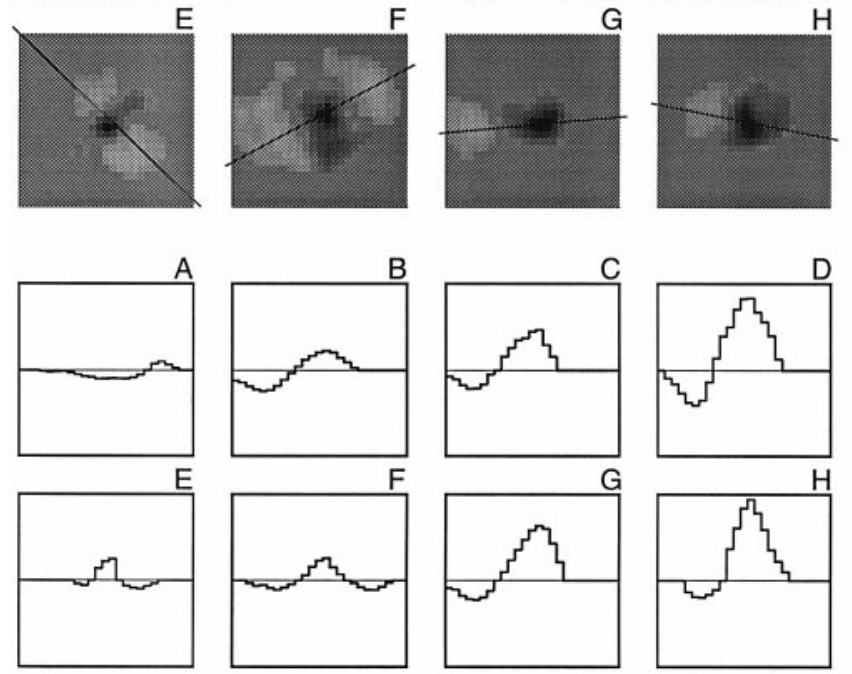

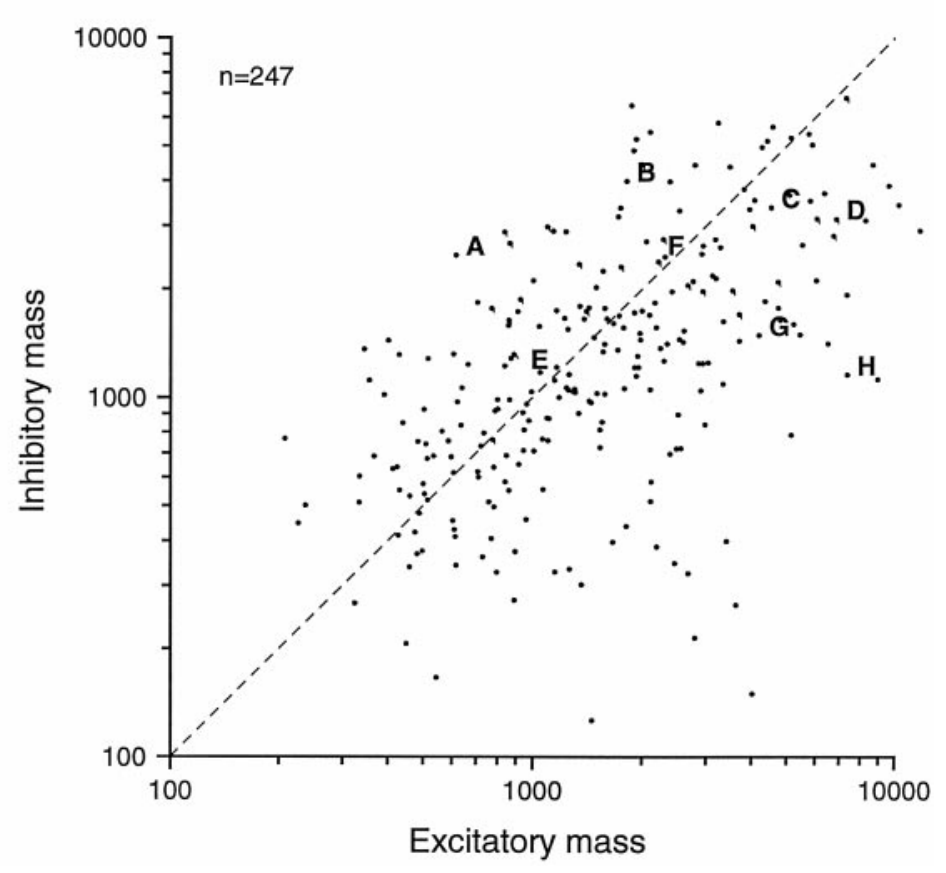

Figure 6. Scatter plot of excitatory and inhibitory RF masses. Excitatory (inhibitory) mass was measured as the integral of the absolute value of excitatory (inhibitory) RF values over the excitatory (inhibitory) area of the RF (see Fig. 4). Mass units are impulses per second per millimeter of stimulus relief (see Results). The dashed line in the scatter plot (right) represents balanced excitatory and inhibitory mass. The sample RFs on the top left ( $A-H$ ) illustrate a wide range of excitatory and inhibitory mass combinations. The line through each RF passes through the excitatory and inhibitory peaks. The plots below the two-dimensional RFs (bottom left), which display the RF values ( $y$-axis) along these lines, are meant to display the relative intensities of the excitatory and inhibitory components. The letter above each RF is keyed to a point in the scatter plot.
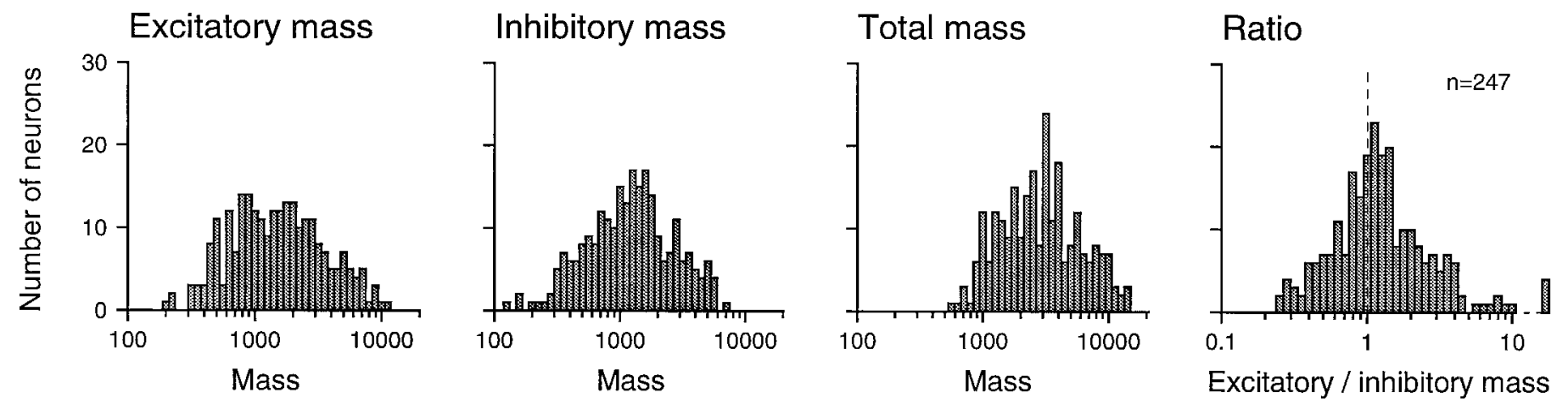

Figure 7. Distributions of RF masses. Axes are as described in Figure 5 except that the $x$-axis represents mass rather than area. Mass units are impulses per second per millimeter of stimulus relief (see Results).

$67 \%$ (164 of 247) of area 3b neurons had larger inhibitory than excitatory areas. The total RF areas ranged from 7.4 to $64 \mathrm{~mm}^{2}$ (mean of $32.3 \mathrm{~mm}^{2}$ ). This mean receptive field area is many times greater than the primary SAI and RA afferent receptive field areas measured with single, scanned dots (Johnson and Lamb, 1981), which indicates a large cumulative divergence between the periphery and area 3b. Larger inhibitory than excitatory RF areas suggest greater inhibitory than excitatory divergence.

\section{RF mass}

The excitatory and inhibitory effects are graded, and therefore a single RF region may have a small or large overall effect on firing rate, depending on the magnitudes of the excitatory or inhibitory weights within its boundaries. Examples of variation in RF values across the skin surface are shown in Figure 6. To summarize this aspect of the RF structure, we integrated these intensities over the excitatory and inhibitory areas and termed the resulting measures excitatory and inhibitory "mass." More precisely, the excitatory (inhibitory) mass was defined as the sum of the positive (negative) RF bin values and has units of impulse per second per millimeter of stimulus relief. The thresholded RF was used for this calculation for the same reasons given for the calculation of RF area.

The distributions of excitatory mass, inhibitory mass, excitatory to inhibitory mass ratio, and total RF mass are shown in Figures 6 and 7. Like the excitatory and inhibitory areas, the area $3 b$ excitatory and inhibitory masses were distributed widely and without any obvious clustering. Both excitatory and inhibitory mass varied by 50:1 across the population, and they were more highly correlated with each other than were the excitatory and inhibitory areas. The minimum, mean, and maximum excitatory 


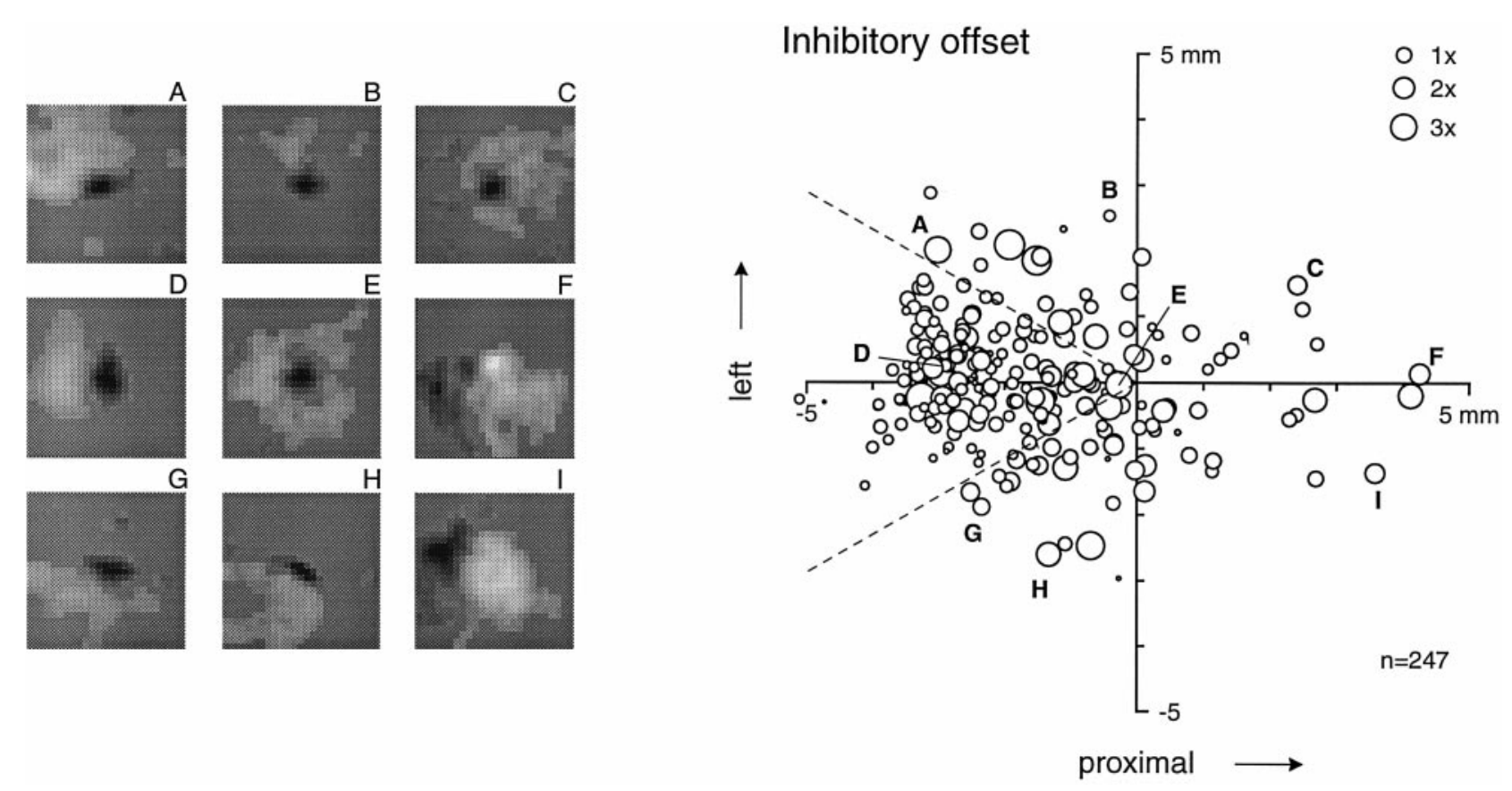

Figure 8. Scatter plot of locations of centers of inhibitory mass relative to centers of excitatory mass. The origin of the graph on the right represents the center of excitatory mass for each neuron. The axes, like the RF images shown throughout this paper (e.g., Fig. 2), are oriented as though the finger was pointing to the left with the glabrous surface down and the RF was viewed through the back of the finger. The $x$-axis represents proximal displacement of the center of inhibitory mass relative to the center of excitatory mass. The $y$-axis represents (anatomical) leftward displacement to the centers of mass. The size of each circle is proportional to the ratio of inhibitory to excitatory mass (see key at top right). The sample RFs on the left (A-I) illustrate examples in which the center of inhibitory mass is displaced distal and leftward $(A)$, leftward $(B)$, etc. The letter above each RF is keyed to a point in the scatter plot.

masses were 210, 2140, and 10,300 mass units, respectively. The comparable values for inhibitory mass were 125, 1620, and 6830 mass units. As seen for area, neither distribution was significantly different from a normal distribution when plotted in logarithmic coordinates $(p>0.20$; SPSS). The bivariate (excitatory and inhibitory mass) log plot shown in Figure 6 has means of 3.176 and $3.080 \log _{10}$ units, SDs of 0.367 and $0.344 \log _{10}$ units, and a correlation of 0.56 . The geometric mean excitatory mass was $25 \%$ larger than was the mean inhibitory mass. The total RF mass (excitatory plus inhibitory) ranged from 590 to 14,200 mass units (mean of 3760 mass units).

Examples of RFs with a range of excitatory and inhibitory masses are shown on the left of Figure 6. Mass differences are illustrated using histograms that show the relative excitatory and inhibitory values in each RF along the lines shown on the RF plots. In general, the peak inhibitory values were less than were the peak excitatory values. As a result, even though the inhibitory areas were typically greater than were the excitatory areas (Fig. $5)$, the excitatory masses were, on average, greater than were the inhibitory masses (Fig. 7). Sixty-one percent (150 of 247) of area $3 \mathrm{~b}$ neurons had larger excitatory than inhibitory masses.

There was no significant correlation between excitatory mass and excitatory area (correlation, $0.11 ; p>0.05$ ); however, inhibitory mass and inhibitory area were correlated (correlation, 0.39; $p<0.01$ ). Excitatory mass was correlated, whereas inhibitory mass was anticorrelated, with the average, evoked firing rate (partial correlation coefficients of 0.85 and -0.17 , respectively). Excitatory and inhibitory areas, in contrast, had little if any relationship to the average, evoked firing rate (partial correlations of 0.00 and 0.08 , respectively). An interesting correlation was observed between the excitatory and inhibitory masses and the responses of the cortical neurons to sustained indentation. A subset of the 247 neurons reported here ( 87 neurons) were studied with sustained indentation $(1000 \mu \mathrm{m})$ using a servo-controlled linear motor and a punctate probe (Chubbuck, 1966). Half of those neurons (44 of 87) responded to the sustained indentation with a sustained impulse rate significantly above the background rate $(p<0.05$; $t$ test; paired difference between rates in $0.5 \mathrm{sec}$ intervals, one just before and one beginning $1.0 \mathrm{sec}$ after the onset of indentation; $1.5 \mathrm{sec}$ duration; 20 repetitions). Neurons with a sustained response to steady indentation had excitatory and inhibitory RF masses that were, on average, twice those of neurons without a sustained response $(p<0.001 ; t$ test $)$. Regression analysis showed that this sustained response was related three to four times more strongly to excitatory than to inhibitory mass.

\section{Relative locations of excitatory and inhibitory RF regions}

Because the relative locations of excitation and inhibition are critical for stimulus selectivity, we determined the spatiotemporal relationship of the centers of excitatory and inhibitory RF mass. The center of excitatory (inhibitory) mass was computed from the thresholded RFs as the weighted mean location of all bins within all excitatory (inhibitory) subregions, with the location of each bin weighted by its absolute excitatory (inhibitory) value. The result of this analysis is shown in Figure 8. The position of each circle in this plot represents the position of the center of all inhibitory RF mass relative to the position of the center of all excitatory RF mass for a single neuron. The area of each circle represents the ratio of inhibitory to excitatory mass.

The dispersion of points around the center of Figure 8 indicates that, across the population, the inhibitory center of mass of 

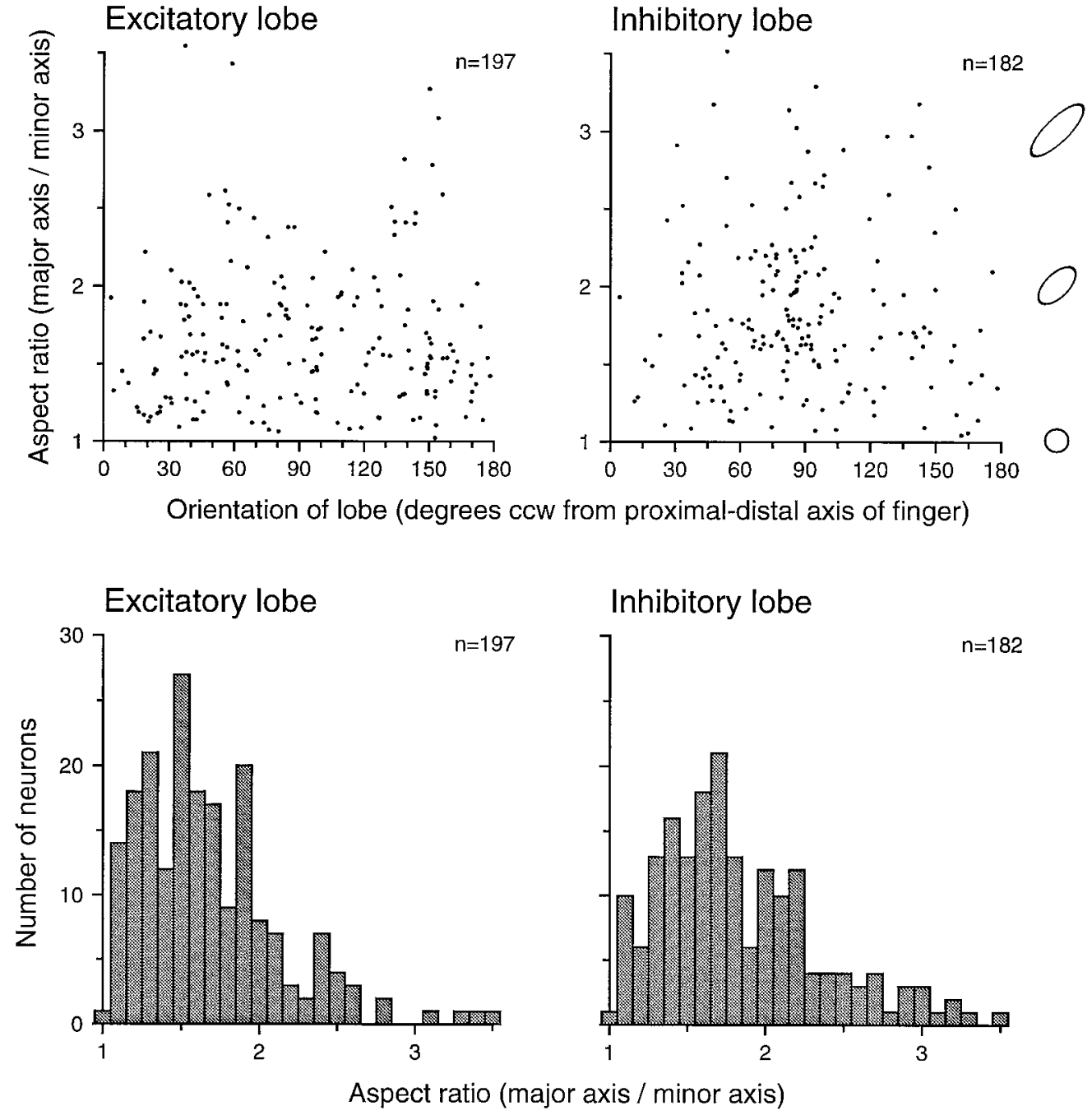

Figure 9. Elongation and orientation of dominant excitatory and inhibitory RF lobes. Dominant lobes [defined as continuous regions of RF excitation (inhibition) that contained at least $80 \%$ of the total excitatory (inhibitory) mass] were fitted with a twodimensional Gaussian function to obtain ellipsoidal descriptors of orientation and elongation (see Results). Top row, The $x$-axis of each graph represents the angle of the lobe's major axis relative to the finger axis. The $y$-axis represents the ratio of the lengths of the best-fitting ellipsoidal major and minor axes. Ellipses at the right illustrate aspect ratios of 1.0, 2.0, and 3.0. Bottom row, Distributions of excitatory and inhibitory aspect ratios are shown. The $x$-axis represents the aspect ratios of the excitatory and inhibitory lobes. The $y$-axis values represent the number of neurons with each aspect ratio. the RF was found at all positions around the excitatory center of mass. This is also illustrated in the examples on the left of Figure 8. However, it is evident that the center of inhibitory mass was displaced toward the fingertip relative to the excitatory center of mass in most RFs. Seventy-two percent of all points in Figure 8 fell in a wedge $60^{\circ}$ wide to the left of the origin (between the dashed lines). The most intense concentration was $2.5-3.0 \mathrm{~mm}$ distal to the excitatory center of mass. The fact that $72 \%$ of the centers of mass fell within this wedge does not mean that most of the inhibition fell within this region; only 36\% (89 of 247) of the RFs had most of their inhibitory mass located in this wedge (see Fig. 3).

Because the stimulus was scanned in the proximal-to-distal direction at $40 \mathrm{~mm} / \mathrm{sec}$, a $2.5-3.0 \mathrm{~mm}$ distal offset also corresponds to inhibition with a time lag of $65-75 \mathrm{msec}$ relative to the peak of excitation. Although a temporal lag seems like the most likely explanation for this inhibitory offset, a fixed time lag of 65-75 msec would result in a spatial offset that was proportional to scanning velocity. However, other data from these same neurons show that this offset is unaffected by changes in scanning velocity from 20 to $80 \mathrm{~mm} / \mathrm{sec}$, so the explanation must be more complex (J. DiCarlo and K. Johnson, unpublished observations; see Discussion).

\section{Shape of excitatory and inhibitory RF regions}

The shapes of RF subregions were examined because they may indicate neuronal selectivity for certain tactile forms. Excitatory and inhibitory RF subregions varied in shape from nearly round to highly elongated, as can be seen from the many examples displayed here. To characterize the elongation and orientation of the excitatory and inhibitory subregions, we segmented each RF into distinct lobes (or islands). A lobe was defined as a continuous region of excitation or inhibition in the thresholded RF. It was classified as a dominant excitatory or inhibitory lobe if it contained at least $80 \%$ of the total excitatory or inhibitory mass. Eighty percent (197 of 247) of the area 3b RFs had a dominant excitatory lobe defined in this way, and 74\% (182 of 247) had a dominant inhibitory lobe. The elongation and orientation of each dominant lobe were measured by fitting its distribution of excitatory (or inhibitory) weights with a bivariate Gaussian density function. Elongation was defined as the ratio of major to minor SDs along the principal axes of the Gaussian function (i.e., aspect ratio). Orientation was defined as the angle of the major axis counterclockwise from the proximal-distal finger axis. The results are displayed in Figure 9. The majority of dominant excitatory $(60 \%, 118$ of 197$)$ and inhibitory $(73 \%, 132$ of 182) lobes had aspect ratios $>1.5$. The excitatory lobes were oriented in all 

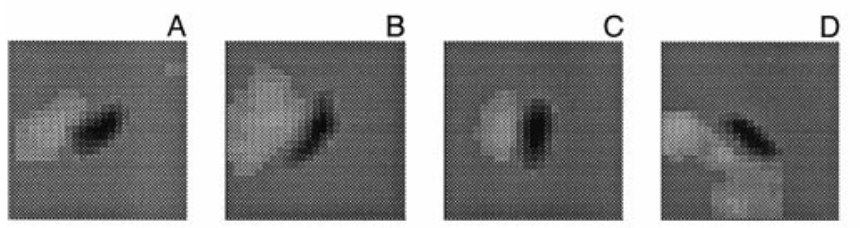

E
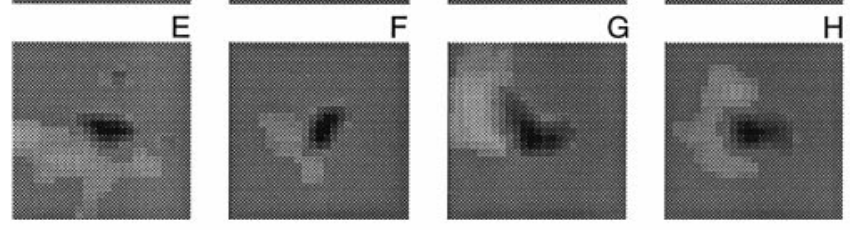

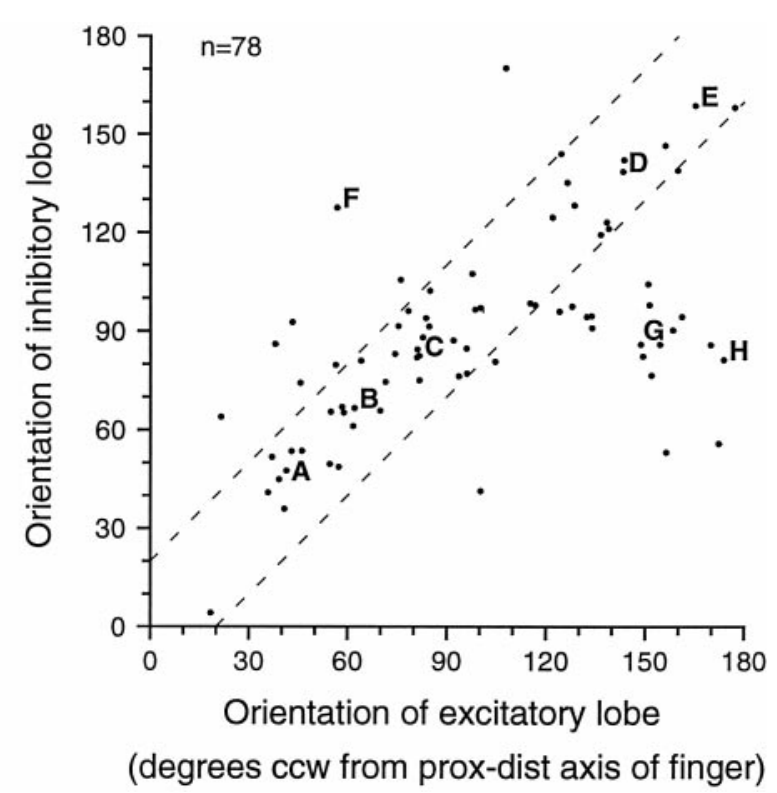

Figure 10. Relationship between excitatory and inhibitory RF lobe orientations. Only neurons with dominant excitatory and inhibitory lobes, both with aspect ratios $>1.5$, were included $(n=78$, see Fig. 9). Excitatory and inhibitory RF lobe orientations tended to be similar (correlation coefficient $=$ 0.586). Sixty-five percent of the points lie within $\pm 20^{\circ}$ of the diagonal (within the dashed lines on the right). Examples are shown on the left $(A-H)$.

directions (i.e., the orientations were not significantly different from a uniform distribution; $p=0.641$; SPSS). However, the inhibitory lobes were preferentially bunched around $90^{\circ}$ counterclockwise from the proximal-distal finger axis, as can be seen in Figure $9(p=0.005$; SPSS $)$.

The shape of the excitatory and inhibitory RF regions of a neuron might confer some stimulus selectivity (e.g., to elongated bars), but the relationship between excitatory and inhibitory RF regions could confer even greater selectivity. We examined the relationship between excitatory and inhibitory lobes in the 78 neurons whose RFs had dominant excitatory and inhibitory lobes with aspect ratios $>1.5$. A majority of these RFs $(65 \%, 51$ of 78$)$ contained dominant excitatory and inhibitory lobes that were aligned to within $20^{\circ}$ of one another (within the dashed lines of Fig. 10). The number expected by chance is 17 of $78(22 \%)$, and the difference is highly significant $(p<0.001)$. In those cases in which the lobes were misaligned, the inhibitory lobe tended to be oriented at or near $90^{\circ}$ counterclockwise from the proximaldistal finger axis relative to the scanning direction. The points labeled $G$ and $H$ in Figure 10 are examples of such fields.

\section{Reliability, goodness-of-fit, and generality}

Although the RFs presented here provided the best linear approximations of the neural responses to the random dot patterns, there are several reasons why the estimated RF for a single neuron might be a poor approximation of the true response properties of the neuron. First, variability in the estimated RF because of variability in the response may be large enough to render the RF estimate unreliable. Second, few relationships in biology are truly linear. A first-order (linear) RF approximation is justified only by the degree to which it captures the repeatable and therefore potentially explainable variation in the responses. Third, even if the RF provides a good description of the neural responses to the random dot patterns, it may not account well for neural responses to other kinds of stimuli. Each of these issues is addressed below.

\section{Reliability}

The reliability of each RF estimate was assessed in two ways. The first method was based on a measure of the noise in each RF estimate. This provided an index of the random pixel-to-pixel variation in the RF estimate and therefore an indication of the variability in each RF relative to other RFs. The second method was based on the correlation between repeated, independent estimates of the same receptive field.

Standard methods of error analysis (Draper and Smith, 1981) provide a basis for estimating the variance of the noise in the weight values (the 625 excitatory and inhibitory values that compose a single RF estimate). The application of these methods to our data suggest that the correlations between errors in adjacent RF bins are low; however, a precise estimate of the RF noise requires precise, bin-by-bin knowledge of the response variance that is difficult to obtain. Instead, we devised a signal-to-noise index based on direct measurement of the variation in RF bin values. Once the RF was estimated, it was filtered with a twodimensional Gaussian filter with a SD $(300 \mu \mathrm{m})$ that was small relative to the spatial dimensions of interest (see Appendix). We proceeded with the assumption that the spatial frequency spectrum of the RF noise was similar across neurons and therefore that this filtering removed the same fraction of the total RF noise variance in each neuron. In that case, the variance of the removed noise (difference between the raw and filtered RFs) is a measure of the total noise in the estimated RF. The magnitude of the RF noise relative to the RF signal is the relevant measure of RF reliability. Thus we adopted the ratio of the $\mathrm{SD}$ of the noise removed by the Gaussian filter to the peak filtered RF value as an index of the reliability of the RF estimate. This measure is referred to as the RF noise index.

Figure 11 shows RFs from seven area 3 b neurons with similarly structured RFs and noise indices ranging from 5 to $35 \%$. This figure shows that as the noise index increases, the raw (unfiltered) $\mathrm{RF}$ becomes more variable, and the thresholded RF starts to 


\section{Receptive field noise standard deviation (\% of signal peak)}
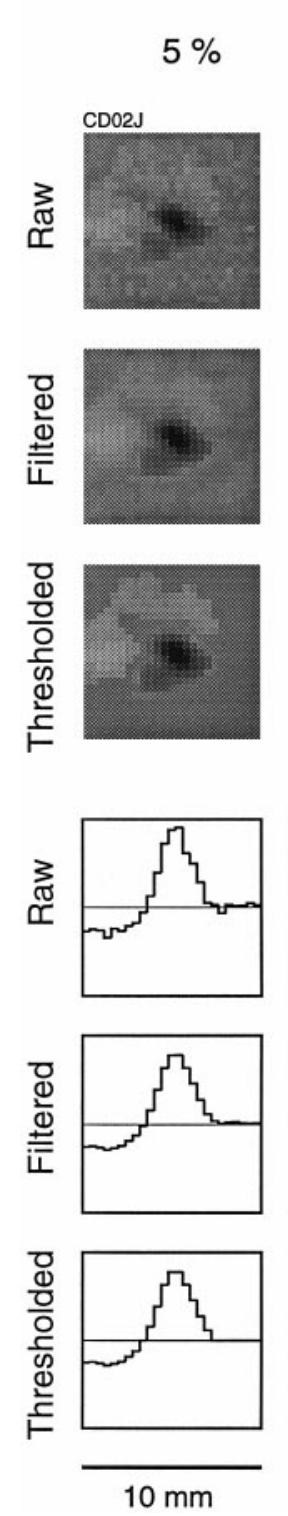

$10 \%$
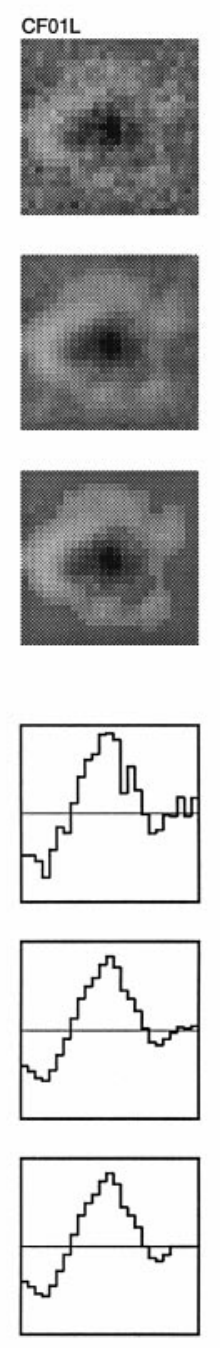

$15 \%$
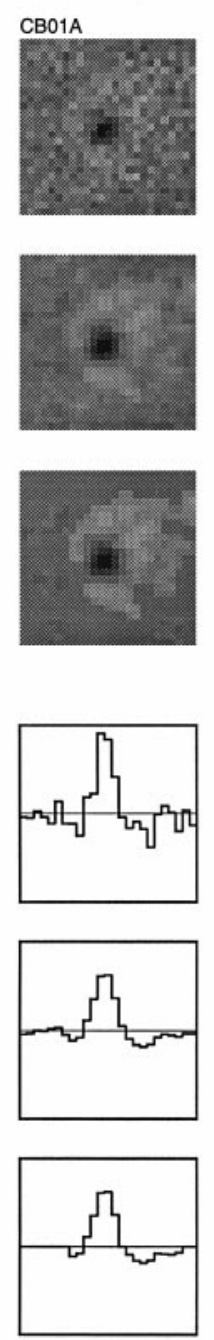
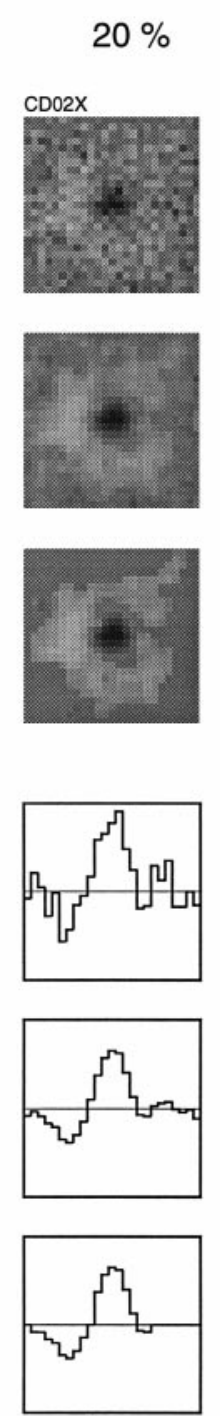
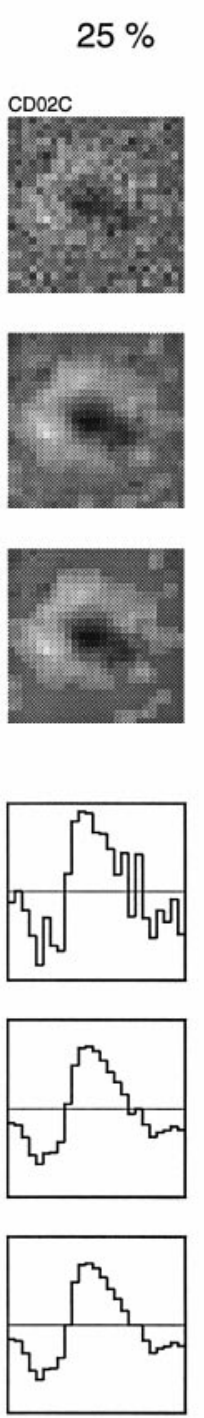
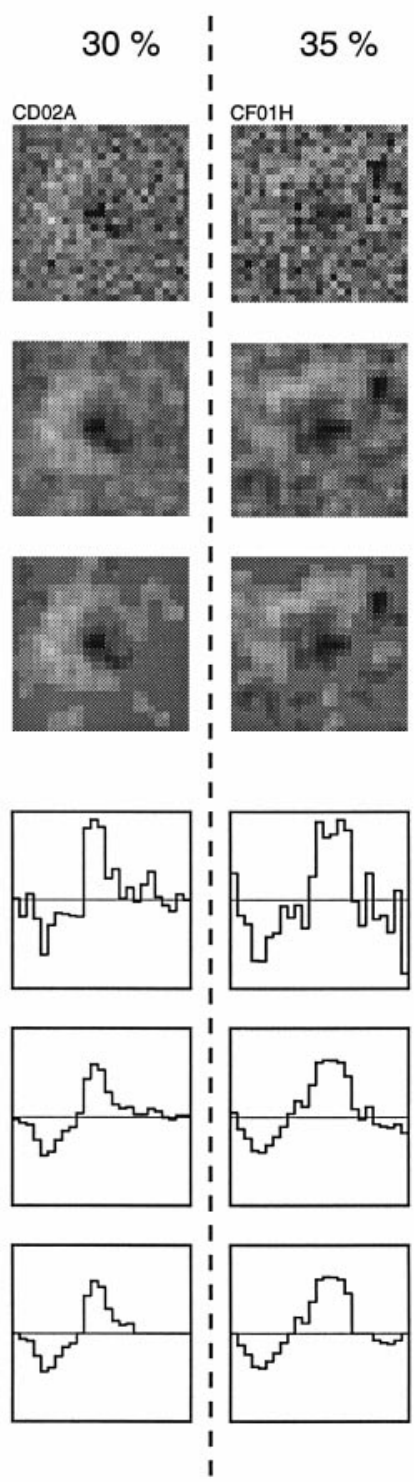

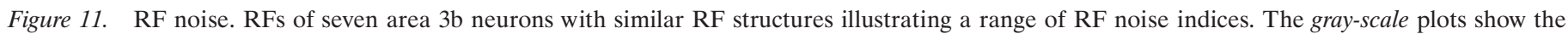

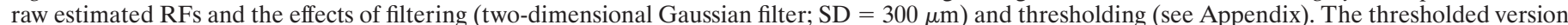

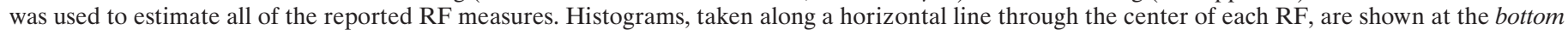

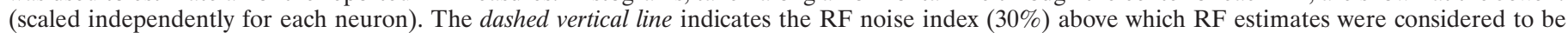
too variable for reliable measurements and were not included in the final analyses.

include regions that may be attributable to noise in the RF estimate. The noise index was, on average, related inversely to the total number of action potentials entering the RF estimate. For example, RF estimates with noise indices between 30 and $35 \%$ were based on recordings whose impulse counts averaged 2500 , which corresponds to an average rate of 4 impulses/sec. Larger numbers of impulses generally produced lower noise indices and less RF variability. For example, all neurons that responded with $>10,000$ impulses to the random dot stimulation (103 of 247 neurons) had noise indices $<30 \%$ (mean of $8.4 \%$ ).

An alternative measure of the reliability of the RF estimates was obtained by dividing the data into two independent sets, obtaining independent RF estimates from the two data sets, and comparing the estimates. The data were divided three different ways. The action potentials were assigned to separate data sets from (1) alternate (odd- and even-numbered) sweeps of the random dot pattern, which were separated by only $200 \mu \mathrm{m}$; (2) the first and last halves of each sweep (duration, $6.25 \mathrm{sec}$ ); and (3) the first $(\sim 50)$ and last $(\sim 50)$ sweeps across the pattern. The three methods of dividing the data assess different possible reasons for a lack of RF repeatability. Division by the first method assesses the effect of noise alone, for there is no other reason that RF estimates obtained from interleaved data should be different. The second and third methods introduce other potential reasons for differences. Both involve estimates from responses evoked by nonoverlapping stimuli. Division by the second method also reveals differences that might be caused by short-term adaptation between the first and second halves of each $6.25 \mathrm{sec}(250 \mathrm{~mm}$ at 


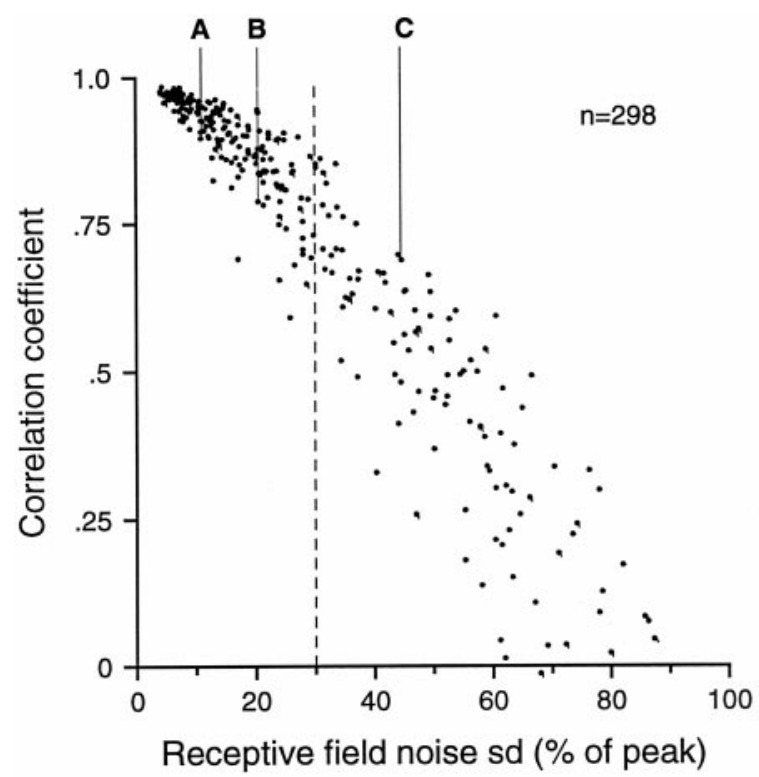

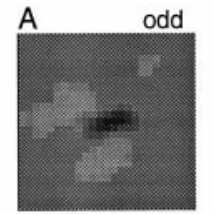
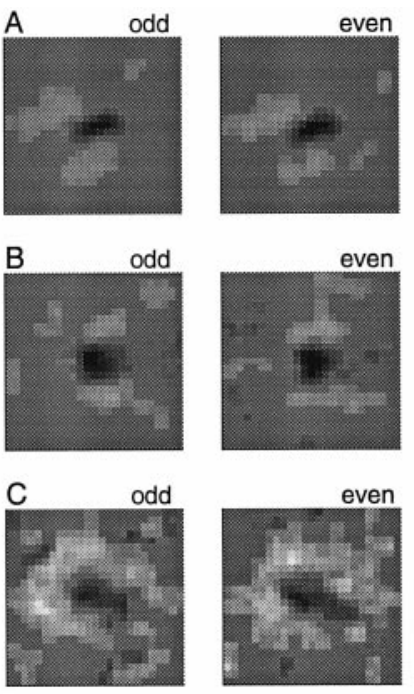

RF noise: $11 \%$

Corr coeff: .90

RF noise: $20 \%$

Corr coeff: .80

RF noise: $45 \%$

Corr coeff: .69

Figure 12. RF repeatability. Examples of three pairs of independent RF estimates from three cortical neurons are shown on the right. Two independent $\mathrm{RF}$ estimates for each neuron were computed from spike data collected on even- and odd-numbered drum scans, respectively (see the Results). The correlation coefficients plotted on the $y$-axis of the graph on the left and displayed to the right of each pair of RFs are correlations of the 625 paired RF weights of the two independent RF estimates from each neuron. The $x$-axis represents the RF noise index displayed in Figure 11. Note that the noise index for each RF in this figure is based on half the data and is thus $\sqrt{ } 2$ greater than the noise index of the RF obtained from all the data. The labels $A, B$, and $C$ above the example RFs are keys to points in the graph on the left. $A$ and $B$ were chosen to illustrate the less repeatable cases among neuronal RFs with noise indices near 10 and $20 \%$, respectively. $C$ was chosen to show how good the repeatability can be even when the noise index is over $40 \%$, which is well above the cutoff (dashed vertical line) used in this study for measuring RF parameters.

$40 \mathrm{~mm} / \mathrm{sec}$ ) sweep across the random dot pattern. Division by the third method reveals differences that might be caused by longerterm changes, because the neuron is subjected to continual stimulation over a period of almost $14 \mathrm{~min}$.

An estimate of the RF and its noise index, as defined above, was obtained from each of the six sets of action potentials (data division in three different ways). Repeatability was assessed by Pearson's correlation between the two independent RF estimates obtained from each of the three divisions. The correlation calculation was effected by plotting the pairs of weights from matching $\mathrm{RF}$ bins in a two-dimensional plot (data not shown) and by calculating the correlation of this bivariate scatter. Results from the first data division method are displayed in Figure 12, which shows that the correlation was, as expected, a declining function of the RF noise index and that RFs with noise indices $<30 \%$ were highly reproducible (mean correlation $=0.893 ; \mathrm{SD}=0.078$ ). This high degree of correlation is not attributable to similarities in the RFs among the population of area $3 \mathrm{~b}$ neurons, because the mean correlation of the RFs of different neurons with RF noise indices $<30 \%$ was only 0.465 . These results and our subjective assessment of data like those illustrated in Figure 11 caused us to adopt an RF noise index of $30 \%$ as the threshold for inclusion in the study. Examples of RFs with 11, 20, and $45 \%$ noise indices in Figure 12 show that this is a conservative threshold. The examples at 11 and $20 \%$ illustrate the less repeatable cases (lower correlations) at each of those noise indices. In contrast, the example at $45 \%$ was chosen to illustrate how reliable RF estimates with high noise indices can be.

When examining the RF pairs in Figure 12, it should be noted that they are each based on only half the data that went into the RF estimates for each neuron. The noise indices for each point in the scatter plot are the average of the noise indices obtained from the two RFs computed from the interleaved data sets and are thus larger than the noise indices of the RFs computed using the full data set (by approximately a factor of $\sqrt{2}$ ). The plot in Figure 12 is intended mainly to show the relationship between the measure that we used as an index of the variability of the RF estimate (noise index) and a direct measure of RF variability (the correlation between repeated measures).

The second and third methods of segregating the data resulted in two more correlation coefficients for each neuron. These correlation coefficients were almost identical to the correlation coefficients based on the interleaved data division (Fig. 12), and plots of these correlation coefficients versus the noise index were indistinguishable from Figure 12. Pairwise analysis of correlation coefficients from single neurons showed a slight drop relative to the correlations based on the interleaved data. The correlations based on the first and last 50 sweeps (division by method three) were, on average, 0.041 correlation units lower than were the correlations based on interleaved sweeps. The correlations based on the first and last halves of each 6.25 sec sweep (division by method two) were, on average, 0.082 units lower than were the correlations based on interleaved sweeps. We attribute these small differences to the fact that the first correlation coefficient was based on independent responses to the same dot pattern, whereas the latter two correlations were based on RFs obtained from responses to different random dot patterns.

\section{Goodness of fit}

A brief description of our approach to measuring goodness-of-fit follows; a complete description is given in Appendix. The goal of the analysis is to determine how well the actual neural response is described by the response predicted by the linear RF (see Fig. $2 C-E$ ). The neural response to any stimulus consists of two parts, a random part (noise) and a repeatable part, which is, in principle, explainable and is the part we aim to understand. The 


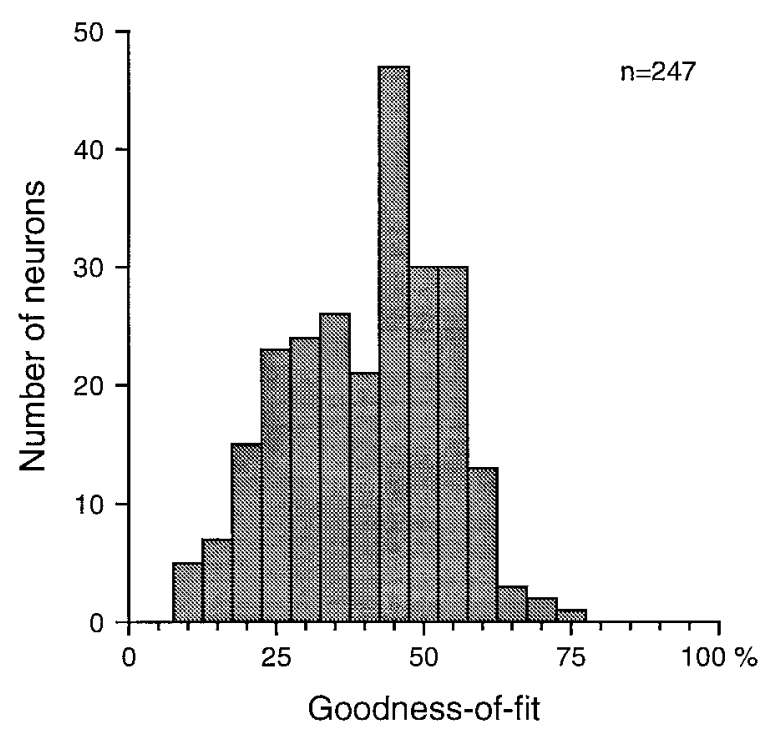

Figure 13. Distribution of goodness-of-fit. Goodness-of-fit was defined as the fraction of the explainable neural response variance (i.e., not including noise variance) that was captured by the predicted response (response rates predicted by the linear RF). See Appendix for details.

repeatable or explainable part is, in turn, composed of two parts, a part that can be explained as the sum of the excitatory and inhibitory contributions described by the RF (the linear part) and the remainder, which is by definition the nonlinear part. The goodness-of-fit measure presented here is the fraction of the repeatable or explainable variance that is, in fact, explained by the summation effects specified by the RF. The regression method provides a measure of the variance explained by the RF (variance of the linear part). All that remains to measure goodness-of-fit is an estimate of the repeatable (linear plus nonlinear) variance.

The repeatable part of the neural response can be estimated as the mean of many repeated trials, but this is impractical when a single trial requires almost 14 min of stimulation. However, the noise variance can be estimated accurately from only two trials, which provides an effective way of estimating the variance of the repeatable part. By definition, the noise and repeatable variations are uncorrelated, so the variance of the repeatable part is the difference between the total response variance and the noise variance. The goodness-of-fit measure is the percent of this "explainable" variance that was actually explained by the estimated RF.

The distribution of goodness-of-fit values is shown in Figure 13. A goodness-of-fit value of $100 \%$ indicates that the RF described the repeatable component of the neural response completely. A value of $0 \%$ indicates that the RF explained none of the repeatable part. Figure 13 shows that the distribution of goodness-of-fit values for area $3 b$ neurons was approximately normal and unimodal, indicating no clear distinction between neurons that have predominantly linear and those that have predominantly nonlinear responses. The mean and SD of the distribution were 40.3 and $13.3 \%$, respectively. For comparison, note that the goodness-of-fit of the RF shown in Figure 2 was $53 \%$. The mean of the distribution in Figure 13 indicates that, on average, area $3 \mathrm{~b}$ neurons are moderately well described by linear RFs (the mean $40.3 \%$ corresponds to a correlation of 0.63 between the predicted and explainable response), but the breadth of the distribution suggests a wide range of degrees of response linearity.

\section{Generality}

How do RFs estimated with one set of stimuli account for the responses to other kinds of stimuli? Unlike repeatability and goodness-of-fit, generality is not amenable to a complete analysis because there are infinitely many other stimuli. As a first step, we examined the neural responses to scanned, raised letters because they contain stimulus elements not well represented in the random dot pattern (e.g., edges and corners) and because they have been used in previous psychophysical (Vega-Bermudez et al., 1991) and neurophysiological (Phillips et al., 1988; DiCarlo et al., 1994; Johnson et al., 1995; Hsiao et al., 1996) studies. Although a quantitative analysis of the neural responses to the letter stimuli is beyond the scope of this paper, responses of different neurons are usually qualitatively different from one another (Fig. 14; Bankman et al., 1990), so that visual inspection of the responses is informative. For example, Figure 14 shows the responses of five area $3 \mathrm{~b}$ neurons to the letter stimuli, the RFs determined with the random dot stimulus and the responses to letter stimuli predicted by these RFs. These examples are representative of 212 area $3 \mathrm{~b}$ neurons studied with both letters and random dots. In some cases, the predicted responses were very close approximations to the observed responses (e.g., Fig. 14A,B), whereas in other cases, the predicted responses matched the observed responses poorly (e.g., Fig. 14C). In most cases, the match was qualitatively similar but clearly not perfect (e.g., Fig. 14D,E).

\section{DISCUSSION}

The principal aim of this study was to obtain a characterization of the RFs underlying the response properties of neurons in area $3 \mathrm{~b}$ representing the glabrous distal fingerpads. Of the 330 neurons studied, 247 yielded highly reliable estimates of their RF structures.

The most significant finding was the diversity of patterns of RF excitation and inhibition. However, within this diversity, there were certain unifying themes. Most neuronal RFs had a single, central region of excitation that contained $>80 \%$ of the excitatory mass. In $78 \%$ of the RFs, this was paired with a contiguous, single region of inhibition that lay on one side of the excitation $(52 \%)$, surrounded it partially ( $23 \%$ ), or, more rarely, surrounded it fully (3\%). Among the remaining $22 \%$ in which the inhibition did not form a single, continuous region, the majority (15\%) had two regions of inhibition on opposite sides of the excitation. A significant feature of all of the RFs in this study was an overall distal displacement of the inhibitory subfields relative to the excitatory center (Fig. 8), which coincided with the scanning direction.

The diversity in RF structure was matched by wide diversity in $\mathrm{RF}$ areas and masses. The total RF areas varied almost 10 -fold, and the total RF masses varied almost 25-fold. Overall, the excitatory and inhibitory influences on the neural responses were nearly balanced. A majority of neurons (67\%) had larger inhibitory than excitatory areas, but a majority (61\%) had greater excitatory than inhibitory masses.

\section{Previous studies of RF structure}

Previous studies indicate strong, overlapping excitatory and inhibitory drive throughout the RFs of area $3 \mathrm{~b}$ neurons. Administration of GABA antagonists increases excitatory RF size severalfold (Dykes et al., 1984; Alloway and Burton, 1991), demonstrating that excitatory drive extends over areas ordinarily dominated by inhibition. Conversely, intracellular recordings (Andersson, 1965; Whitehorn and Towe, 1968; Innocenti and Manzoni, 1972) and conditioning stimuli (Laskin and Spencer, 

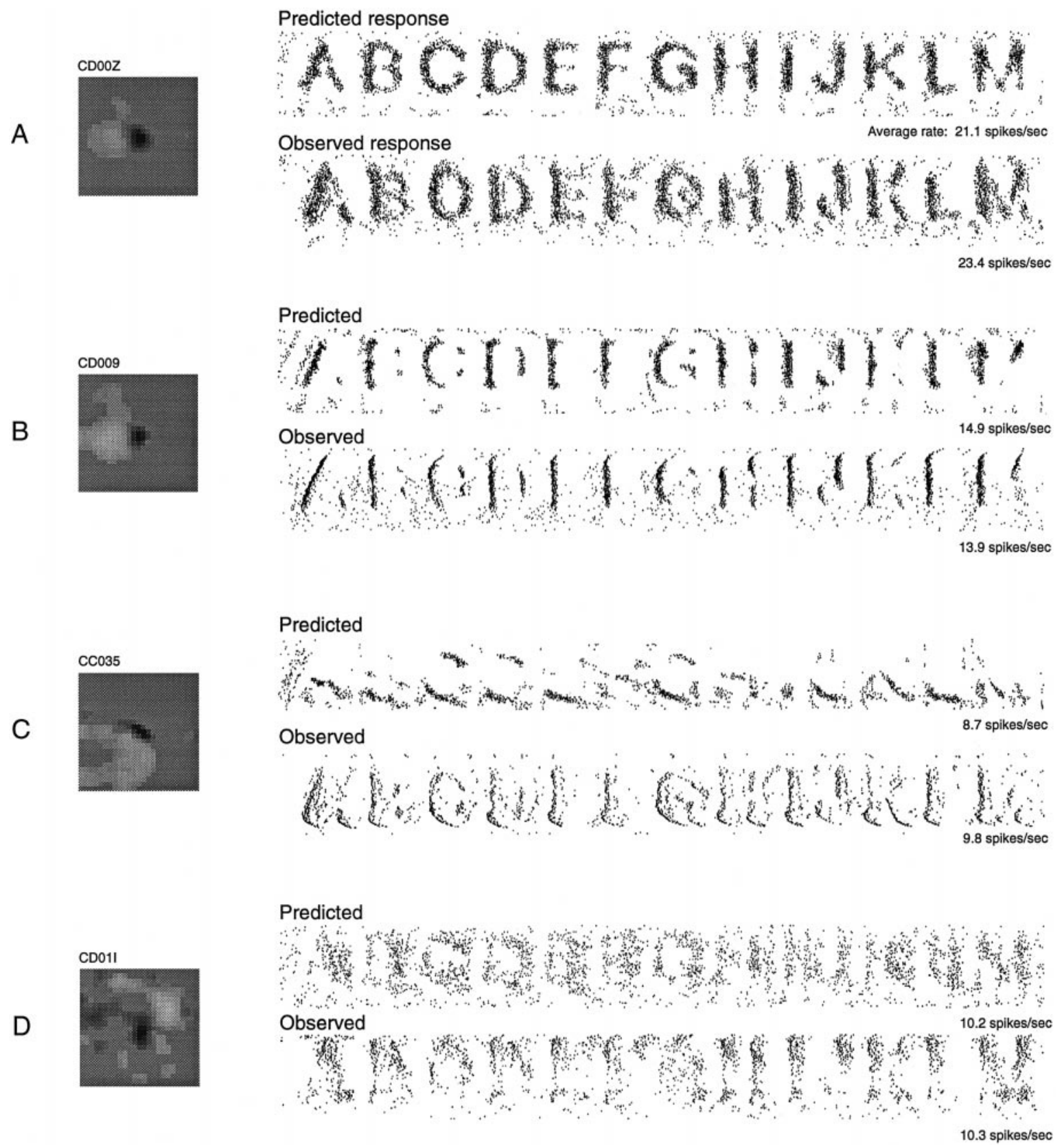

\section{Predicted}
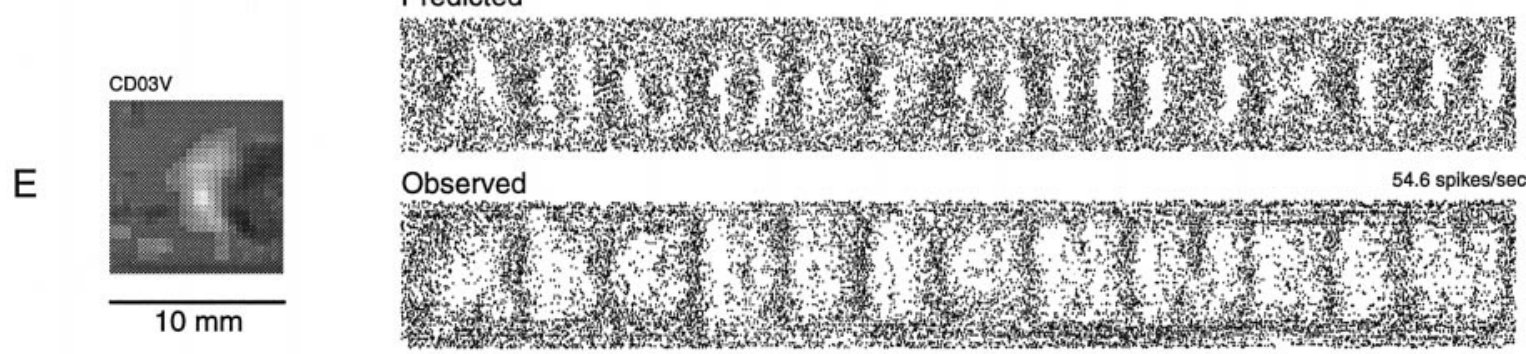

$\overline{10 \mathrm{~mm}}$

49.1 spikes/sec

Figure 14. Generality of the RFs. The responses of five area $3 \mathrm{~b}$ neurons (SEPs) studied with both random dots and embossed letters. The predicted responses to the embossed letters were determined by convolving the RF (left) determined from the response to the random dot pattern with a template of the letter stimulus. Predicted firing rates were converted into action potentials using a Poisson spike generator, with the overall predicted spike rate adjusted to coincide with the observed spike rate. The predicted and observed responses (right) are in qualitatively good agreement in some cases (e.g., $A, B)$, moderate agreement in others (e.g., $D, E$ ), and only mild agreement in others (e.g., $C$ ). The degree to which these RFs predicted the responses to random dots (the goodness-of-fit measures displayed in Fig. 13 ) were $A, 58 \% ; B, 51 \% ; C, 43 \% ; D, 29 \%$; and $E, 38 \%$. 
1979; Gardner and Costanzo, 1980b,c) demonstrate inhibitory drive throughout the excitatory region. Strong inhibitory as well as excitatory drive has also been shown in the dorsal column nuclei (Andersen et al., 1964a; Jänig et al., 1977) and the thalamus (Andersen et al., 1964b; Iwamura and Inubushi, 1974; Jänig et al., 1979). Nonetheless, previous studies and our own experience have shown that inhibitory RF subregions in area $3 \mathrm{~b}$ neurons on the fingerpads are rarely revealed with a punctate probe (Mountcastle and Powell, 1959; Sur, 1980; Iwamura et al., 1983; Sur et al., 1984). For example, Sur (1980) reported that area 3b neurons on the fingers have only homogenous excitatory RFs. We believe that this major difference between previous findings and the results of the present study is that, in general, the inhibition reported here is only revealed by the suppression of driven activity, which requires stimulation of at least two locations within the RF (Gardner and Costanzo, 1980c). Because we used a stimulus that probed each RF at several locations simultaneously and tested a large number of such combinations, we found significant inhibition in $99 \%$ of the neural RFs studied.

The RF areas reported here are consistent with a general increase in RF area occurring at successive stages of processing in the somatosensory system (Talbot et al., 1968; Robinson and Burton, 1980; Sur, 1980; Sur et al., 1980, 1985). We used exactly the same methods to study the RFs of primary SAI and RA afferents (DiCarlo and Johnson, unpublished observations). The smallest RFs in area $3 b$ were as small as the smallest primary afferent RFs, but the mean and maximum area $3 \mathrm{~b}$ receptive field areas were 2.5 times larger than the mean and maximum primary afferent RF areas. The RF areas reported here, like those in the literature (Mountcastle and Powell, 1959; Hyvarinen and Poranen, 1978b; Sur et al., 1980, 1984, 1985; Recanzone et al., 1992), ranged from areas much smaller than a fingerpad to areas involving most of a fingerpad. Sur and colleagues (Sur et al., 1985) reported that RF areas were smallest in layer IV of area $3 \mathrm{~b}$ and larger in supra- and infragranular layers. We observed similar laminar effects on RF excitatory areas (DiCarlo and Johnson, unpublished observations).

Most area 3b RFs described here have one or two inhibitory regions flanking a region of excitation and are not unlike RFs reported in primary visual and auditory cortices (Jones and Palmer, 1987; Shamma et al., 1993). In primary visual cortex, many neurons have RFs consisting of a region of excitation and surrounding inhibition or, more frequently, flanking inhibition (Hubel and Wiesel, 1962, 1968; Jones and Palmer, 1987; Palmer et al., 1991; DeAngelis et al., 1993, 1995). In primary auditory cortex, neurons have RFs in "frequency space" consisting typically of a region of excitation with inhibition surrounding the excitation or offset to either side (Shamma et al., 1993). Whether area $3 \mathrm{~b}$ neurons perform the tactile equivalent of the functions performed in the visual and auditory systems is not known.

\section{Response modality}

Current evidence (Johnson and Hsiao, 1992) suggests that primary SAI afferents are responsible for information about form (Phillips and Johnson, 1981) and texture (Blake et al., 1997a), whereas RA afferents are responsible for information about skin motion, including the detection of flutter (Mountcastle et al., 1972; Ochoa and Torebjörk, 1983), slip (Johansson and Westling, 1987; Srinivasan et al., 1987), and minute surface features (LaMotte and Whitehouse, 1986). If this functional distinction is true, then the primary afferent modality that ultimately drives each cortical neuron provides a clue to its function.
Many previous studies have classified those area $3 \mathrm{~b}$ neurons that respond to steady indentation of a punctate probe with a sustained discharge as slowly adapting (SA) and those that respond only to indentation onset and offset as rapidly adapting (RA) (Mountcastle and Powell, 1959; Powell and Mountcastle, 1959b; Paul et al., 1972; Hyvarinen and Poranen, 1978b; Sur et al., 1984; Chapman and Ageranioti-Belanger, 1991). A problem with this classification is that it leads too easily to the assumption that "SA" neurons (i.e., sustained change in firing rate) in area $3 b$ are driven by primary SAI mechanoreceptive afferents and that "RA" neurons are driven by primary RA mechanoreceptive afferents. A neuron driven solely by activity that originates in primary SAI afferents could yield only transient responses to indentation and withdrawal because of feed-forward inhibitory projections (Andersson, 1965). Sur et al. (1984) have advanced this as an explanation for their observation that neurons in cortical layers above and below layer IV yield only transient responses, even in regions where the neurons in layer IV yield sustained responses. Conversely, a neuron driven exclusively by primary RA afferents could yield a sustained response throughout the period of indentation because the animal's attention is drawn to the stimulus, which has an excitatory effect on neurons in area 3b (Hsiao et al., 1993). Thus, whether a cortical neuron responds to steady indentation or not may depend as much or more on central mechanisms as on whether it is driven by primary SAI afferents, RA afferents, or both. For these reasons, we believe that the terms SA and RA should not be used to classify cortical responses until a reliable method for linking those responses to primary SAI and RA afferents is found. We suggest the terms "sustained" if there is a sustained response anywhere in the RF and "transient" otherwise.

We found that approximately half of area $3 b$ neurons had sustained responses (see Results, RF mass). The only statistically significant relationship between this classification and the RF measures we examined was that the RF masses of sustained neurons were, on average, twice those of transient neurons. This result is consistent with the hypothesis that a sustained response is strongly determined by central mechanisms. The relationship to excitatory RF mass cannot be attributed to the response properties of primary SAI and RA afferents because RA afferents are just as responsive to raised dot patterns as are SAI afferents (Johnson and Lamb, 1981; Connor et al., 1990, Connor and Johnson, 1992) and their excitatory masses, when subjected to random dot analysis, are slightly larger on average (5830 vs 4705; DiCarlo and Johnson, unpublished observations).

\section{Mechanisms}

The widely varying patterns of RF excitation and inhibition reported here are likely attributable to varying patterns of subcortical (Andersen et al., 1964b) and cortical (Alloway and Burton, 1991) excitatory and inhibitory convergence. Nothing in this study allows us to distinguish cortical from subcortical mechanisms; however, intracellular cortical recordings (Andersson, 1965; Whitehorn and Towe, 1968; Innocenti and Manzoni, 1972), laminar differences in response properties (Sur et al., 1984, 1985) (DiCarlo and Johnson, unpublished observations), and the effects of cortically applied inhibitory blocking agents (Alloway and Burton, 1991) indicate that cortical processes play a major role in the construction of area $3 \mathrm{~b}$ RFs.

A finding that requires further explanation is the distal offset (i.e., in the scanning direction) of the center of inhibitory mass relative to the center of excitatory mass in many neurons. Thirty- 
six percent of area $3 \mathrm{~b}$ neurons had RFs that contained a single region of inhibition that was offset distally from the region of excitation by $2-3 \mathrm{~mm}$ (Fig. $3 A$ ), and many RFs with more complex regions of inhibition had their centers of inhibitory mass displaced distally relative to their centers of excitatory mass (Fig. 8). Two mechanisms may have contributed to this distal offset. The first is skin mechanics and the response properties of primary afferents, which respond more vigorously to the leading edge of a stimulus (LaMotte and Srinivasan, 1987; Blake et al., 1997b) and to the first dot in a group of raised dots (Johnson and Lamb, 1981). To examine this, we studied 10 primary SAI afferents and 10 primary RA afferents with exactly the same methods used to study the cortical neurons (DiCarlo and Johnson, unpublished observations). Primary RA afferent RFs exhibited little or no "inhibition." Primary SAI afferents had RFs containing a relatively weak "inhibitory" component that trailed the excitation just like a subset of the neurons in area $3 \mathrm{~b}$. However, the strength of this "inhibitory" component was always less than that typically observed in area $3 \mathrm{~b}$ RFs, which suggests that the trailing inhibition observed in area $3 \mathrm{~b}$ is attributable, at least in part, to central mechanisms.

A second factor that could have contributed to the distal shift of the center of inhibitory mass relative to the center of excitatory mass was a temporal delay between RF inhibition and excitation. Studies of the timing of inhibition relative to excitation within the lemniscal pathway, whether that of IPSPs relative to EPSPs (Andersson, 1965) or of "in-field" inhibition (inhibition that spatially overlaps the excitation) (Laskin and Spencer, 1979; Gardner and Costanzo, 1980b,c), report a delay of inhibition relative to excitation. Although temporal mechanisms likely underlie the distal offset of inhibition observed in many area 3b RFs (Fig. 8), studies in which scanning velocity and scanning direction were varied show that a portion of the inhibition in the RF is not temporally delayed and that a simple, fixed delay of 50-75 msec (i.e., 2-3 $\mathrm{mm}$ in the RFs reported here) does not explain the data (DiCarlo and Johnson, unpublished observations).

\section{Cortical representation of form and texture}

The presentation of a tactile stimulus to a single fingerpad activates hundreds of primary afferents innervating that fingerpad. The pattern of activity carried by this population of neurons is the initial neural representation or image of the stimulus. As outlined in the introductory remarks, this image is conveyed and processed through subcortical pathways, through area $3 \mathrm{~b}$, and through successively "higher" cortical areas (e.g., SII, insula). We presume that these processing steps lead to higher representations or images, which are the basis of tactile form recognition and texture perception. In this framework, the ascending pathways are regarded as successive stages of an image-processing chain (Johnson, 1980; Bankman et al., 1990; Johnson et al., 1995), and area 3b is a critical link in this chain. The possible roles of the area 3b RFs reported here can be seen by considering two hypotheses about the neural images underlying form and texture perception.

One possibility is that there is no dramatic transformation of the form of the neural representation of an object. In other words, the final neural representation on which form recognition is based is essentially somatotopic, that is, the primary significance of the activity of a single neuron (pixel in the image) is the presence of a stimulus at a particular skin location. Secondarily, each neuron might signal the presence of a particular spatial feature like an edge or a corner. In other words, each neuron may function as a matched spatial filter used to enhance local spatial features; it is maximally excited by the local spatial pattern configuration on the skin that best matches its excitatory and inhibitory RF structure. Because there are hundreds of neurons in area $3 b$ for each primary afferent (Darian-Smith and Kenins, 1980; Rockel et al., 1980), it is possible that the brain applies a large number of highly selective analyzers to every point in the primary image. In this scheme, a single, somatotopic primary neural image is replaced by many relatively somatotopic images, each specialized to amplify a certain kind of spatial feature. Combinations of one or more of these images might underlie different aspects of tactile perception (e.g., form, roughness, softness, direction of motion, etc.).

A second hypothesis is that the final image is not somatotopic but that it is a highly transformed representation with properties that enable pattern recognition (e.g., translation and size invariance). In this scheme, neural processing in area $3 \mathrm{~b}$ is part of a stagewise process that transforms the initial somatotopic representation into a final, nonsomatotopic representation. For example, a simulated network designed to conform to known rules of connectivity in the somatosensory system and to effect a useful transformation reveals that RF structures at an intermediate stage of the transformation are like many of the RFs reported in this paper (Bankman et al., 1990). That is not to suggest that the somatosensory pathways are structured like this model network but rather that the area $3 \mathrm{~b}$ RF structures are of the kind that would be expected at an intermediate stage in a network effecting a stepwise transformation to a completely altered form of representation.

Whichever of these possibilities is true, the RFs reported here are a clue to the mechanisms responsible for tactile form and texture perception. The similarity of RFs in primary somatosensory, visual, and auditory cortices could mean that the three systems are constructing compatible representations of the external world of the kind that would facilitate sensory integration.

\section{APPENDIX \\ Computation of the RF}

The RF of each neuron is a linear approximation of the effects of stimulus elements (dots) inside the RF on the response of that neuron. That is, the net effect of any portion of the random dot pattern on the response of the neuron is assumed to be the sum of the effects of the dots at each of the skin locations. To determine the effect of a dot at each skin location, we divided the skin surface into a grid of 625 subregions (each $400 \times 400 \mu \mathrm{m}$ ) and formally described the response of the neuron as the sum of the effects of each subregion:

$$
\begin{gathered}
r_{\text {predicted }}(n)=b_{0}+b_{1} x_{1}(n)+b_{2} x_{2}(n)+b_{3} x_{3}(n)+\ldots \\
+b_{625} x_{625}(n) \\
n=1,2, \ldots 20000,
\end{gathered}
$$

where $r_{\text {predicted }}(n)$ is the impulse rate predicted in response to the $n$th position of the random dot pattern, $b_{0}$ is the background firing rate, $b_{i}$ is the strength of the effect of a dot in the $i$ th subregion of skin, and $x_{i}(n)$ is the stimulus relief ( 0 or 0.4 , because each dot was $0.4 \mathrm{~mm}$ in relief) at the $i$ th skin subregion when the random dot pattern was in the $n$th position. As the random dot pattern shifts to a new location, the pattern of dots on the skin and thus the stimulus values $\left(x_{i}(n) ; i=1,2 \ldots, 625\right)$ change, but $b_{0}$ and the grid weights $\left(b_{1}, b_{2} \ldots, b_{625}\right)$ remain fixed. The set of weights is intended to describe the neuron's response to any stimulus 
pattern, and we refer to $b_{1}$ to $b_{625}$ as the linear RF (or, simply, the $\mathrm{RF}$ ) of the neuron. We solve for $b_{0}$ to $b_{625}$ by first rewriting Equation $\mathrm{A} 1$ as the matrix equation:

$$
\mathbf{r}_{\text {predicted }}=\mathbf{X} \mathbf{b}
$$

where $\mathbf{r}_{\text {predicted }}$ is a $20,000 \times 1$ vector containing the predicted rates, $\mathbf{b}$ is a $626 \times 1$ vector containing the constant term $b_{0}$ as the first element and $b_{1}, b_{2} \ldots, b_{625}$ as the remaining elements, and $\mathbf{X}$ is a $20,000 \times 626$ matrix with values of 1 in the first column (the constant multiplier) and appropriate stimulus values ( 0 or 0.4$)$ in the remaining columns. The vector $\mathbf{b}$ that minimizes the meansquared difference between $\mathbf{r}_{\text {predicted }}$ and $\mathbf{r}_{\text {observed }}$, the vector of observed discharge rates, is obtained by solving the linear normal equations (Draper and Smith, 1981) $\mathbf{X}^{T} \mathbf{X} \mathbf{b}=\mathbf{X}^{T} \mathbf{r}_{\text {observed }}$ by inverting the stimulus autocorrelation matrix:

$$
\mathbf{b}=\left(\mathbf{X}^{T} \mathbf{X}\right)^{-1} \mathbf{X}^{T} \mathbf{r}_{\text {observed }} .
$$

The method applies to any stimulus and subsumes methods like reverse correlation (de Boer and Kuyper, 1968; Jones and Palmer, 1987) as special cases. The noise in the RF estimate $\mathbf{b}$ depends on the numerical condition of $\mathbf{X}^{T} \mathbf{X}$ as well as the noise in $\mathbf{r}_{\text {observed }}$ (Press et al., 1992), that is, on stimulus selection. One strength of random dot patterns, white noise (Marmarelis and Marmarelis, 1978), M sequences (Sutter, 1987), etc., is that they produce well-conditioned stimulus autocorrelation matrices $\left(\mathbf{X}^{T} \mathbf{X}\right)$.

This regression formulation does not take into account the neuron's threshold nonlinearity and must therefore be modified. The following modification, termed "least squares with zero removal," is based on hundreds of simulations with hypothetical RFs consisting of a variety of patterns of excitation and inhibition. During periods of strong inhibition, the neuron is silent, and an accurate RF description will predict a large negative response, $r_{\text {predicted }}(n)$. However, if data from this period are included, the regression will register the negative prediction as an error (because the observed firing rate was zero) and will distort the estimated RF to reduce these errors. The ideal solution is to remove equations from Equation A1 that correspond to response bins with zero spikes without removing equations that correspond to bins that happen to have zero impulses during the period when the actual rate is positive. The latter requirement occurs because an accurate estimate of the local rate depends on the bins that happen to be empty by chance as well as on the neighboring bins that happen to have more spikes than the actual local rate. We addressed this issue by removing only equations in which the corresponding response bin was zero and the eight spatially surrounding response bins were also zero. We reasoned that the neural response amplitude varies smoothly (relative to the $400 \times$ $400 \mu \mathrm{m}$ bin size) over the stimulus pattern, so that if the count in a bin is zero and the surrounding bins are also zero, it is likely that the underlying response rate is negative or near zero in that central bin. In practice, the predicted values for empty response bins that are surrounded by empty response bins are almost always negative (see Fig. $2 E$ ). Simulations with a wide variety of rules for zero-bin removal and with a wide range of hypothetical combinations of RF excitation and inhibition showed that this simple rule for zero-bin removal (only when the surrounding eight response bins were zero) provided the least distortion of the estimated RF from the true RF. Although the RF solutions were computed using this zero-bin removal method, the goodness-of-fit of the predicted responses was assessed over all response bins (i.e., including zero bins).

\section{RF filtering and thresholding}

Once the grid of RF weights $\left(b_{1} \ldots, b_{625}\right)$ was determined, it was used to estimate the excitatory and inhibitory areas and masses (summed excitatory and inhibitory values). Because the excitatory and inhibitory weights often blended gradually into the background, a method for segmenting real effects from noise in the RF was required. To minimize the noise, we convolved the RF with a Gaussian filter with a SD $(300 \mu \mathrm{m})$ that was small relative to the spatial dimensions of interest. Next, we eliminated (set to zero) all RF bins with values $<10 \%$ of the peak absolute RF value. A similar threshold has been used previously to compute the area and magnitude of primary afferent responses (Johnson and Lamb, 1981; Blake et al., 1997b). However, a 10\% threshold (or any threshold that does not cut too deeply into the real RF) still allows noise to exceed the threshold. To reduce noise effects (particularly on the measurements of area) further, we required that every nonzero bin in the thresholded RF has at least two of the four adjacent bins be nonzero and of the same sign and also that isolated RF regions have areas of at least $0.7 \mathrm{~mm}^{2}$. Otherwise, the bins were set to zero. The resulting RF is referred to as a thresholded RF. This thresholding method, guided by reasoning about what constitutes a plausible physiological result as well as by extensive simulations, yielded excellent repeatability and unbiased estimates of regions of excitation and inhibition in simulated RFs. The effects of this thresholding method can be seen in Figure 11. If only a Gaussian filter and a $10 \%$ threshold had been used, the calculated excitatory and inhibitory areas would both have been $23 \%$ greater, and the excitatory and inhibitory masses would have been 10 and $14 \%$ greater, respectively.

\section{Goodness-of-fit}

The degree to which each RF captured the neuron's response to the random dot pattern, termed "goodness-of-fit," was assessed as the fraction of the explainable response variance $\left(\sigma_{\text {explainable }}^{2}\right)$ that was actually explained by the linear RF ( $\left.\sigma_{\text {explained }}^{2}\right)$ :

Goodness-of-fit $=\frac{\sigma_{\text {explained }}^{2}}{\sigma_{\text {explainable }}^{2}}=\frac{\left[\sigma_{\text {predicted }}^{2}-\left(626 / n_{\text {bins }}\right) \sigma_{\text {noise }}^{2}\right]}{\left[\sigma_{\text {response }}^{2}-\sigma_{\text {noise }}^{2}\right]}$.

The explainable variance is the component of the total response that would be captured by a description of the neuron's response if it accounted for all of the nonlinear as well as linear parts of the stimulus-response relationship; it was estimated as the variance of the observed response minus the variance attributable to noise. The noise variance $\left(\sigma^{2}\right.$ noise $)$ was estimated by treating responses to neighboring drum sweeps as repeated sweeps, although they were actually displaced by $200 \mu \mathrm{m}$. Analysis of 49 area $3 \mathrm{~b}$ neurons in which we obtained the response to two identical sweeps at each pattern location showed that the error introduced by this approximation on the explainable variance averaged $4.07 \%( \pm 2.04 \%)$. The explained variance was estimated as the variance of the response predicted by the linear $\mathrm{RF}\left(\sigma_{\text {predicted }}^{2}\right)$ minus the part of the noise variance that was fitted by the RF estimation procedure. This correction is required because the regression procedure adjusts the RF parameters to fit the noise as well as the deterministic component of the response. The fraction of the noise variance captured by the regression is determined by the ratio of the degrees of freedom in the RF estimate (626) to the degrees of freedom in the data (the number of independent response histogram bin values, $n_{\text {bins }}$; typically 20,000). 


\section{REFERENCES}

Alloway KD, Burton H (1991) Differential effects of GABA and bicuculline on rapidly- and slowly-adapting neurons in primary somatosensory cortex of primates. Exp Brain Res 85:598-610.

Andersen P, Eccles JC, Oshima T, Schmidt RF (1964a) Mechanisms of synaptic transmission in the cuneate nucleus. J Neurophysiol 27:1096-1116.

Andersen P, Eccles JC, Sears TA (1964b) The ventrobasal complex of the thalamus: types of cells, their responses and their functional organization. J Physiol (Lond) 174:370-399.

Andersson SA (1965) Intracellular postsynaptic potentials in the somatosensory cortex of the cat. Nature 205:297-298.

Bankman IN, Johnson KO, Hsiao SS (1990) Neural image transformation in the somatosensory system of the monkey: comparison of neurophysiological observations with responses in a neural network model. Cold Spring Harb Symp Quant Biol 55:611-620.

Blake DT, Johnson KO, Hsiao SS (1997a) Neural coding mechanisms in tactile pattern recognition: the relative contributions of slowly and rapidly adapting mechanoreceptors to perceived roughness. J Neurosci 17:7480-7489.

Blake DT, Hsiao SS, Johnson KO (1997b) Monkey cutaneous SAI and $\mathrm{RA}$ responses to raised and depressed scanned patterns: effects of width, height, orientation, and a raised surround. J Neurophysiol 78:2503-2517.

Chapman CE, Ageranioti-Belanger SA (1991) Discharge properties of neurons in the hand area of primary somatosensory cortex in monkeys in relation to the performance of an active tactile discrimination task. Exp Brain Res 87:319-339.

Chubbuck JG (1966) Small motion biological stimulator. Johns Hopkins APL Tech Digest May-June:18-23.

Connor CE, Johnson KO (1992) Neural coding of tactile texture: comparisons of spatial and temporal mechanisms for roughness perception. J Neurosci 12:3414-3426.

Connor CE, Hsiao SS, Phillips JR, Johnson KO (1990) Tactile roughness: neural codes that account for psychophysical magnitude estimates. J Neurosci 10:3823-3836.

Darian-Smith I, Kenins P (1980) Innervation density of mechanoreceptive fibers supplying glabrous skin of the monkey's index finger. J Physiol (Lond) 309:147-155.

de Boer E, Kuyper P (1968) Triggered correlation. IEEE Trans Biomed Eng 15:169-179.

DeAngelis GC, Ohzawa I, Freeman RD (1993) Spatiotemporal organization of simple-cell receptive fields in the cat's striate cortex. I. General characteristics and postnatal development. J Neurophysiol 69:1091-1117.

DeAngelis GC, Ohzawa I, Freeman RD (1995) Receptive-field dynamics in the central visual pathways. Trends Neurosci 18:451-458.

DiCarlo JJ, Hsiao SS, Johnson KO (1994) Transformation of tactile spatial form within a cortical column in area $3 b$ of the macaque. Soc Neurosci Abstr 20:567.9.

DiCarlo JJ, Lane JW, Hsiao SS, Johnson KO (1996) Marking microelectrode penetrations with fluorescent dyes. J Neurosci Methods 64:75-81.

Draper N, Smith H (1981) Applied regression analysis. New York: Wiley.

Dykes RW, Landry P, Metherate RS, Hicks TP (1984) Functional role of GABA in cat primary somatosensory cortex: shaping receptive fields of cortical neurons. J Neurophysiol 52:1066-1093.

Gardner EP, Costanzo RM (1980a) Spatial integration of multiple-point stimuli in primary somatosensory cortical receptive fields of alert monkeys. J Neurophysiol 43:420-443.

Gardner EP, Costanzo RM (1980b) Temporal integration of multiplepoint stimuli in primary somatosensory cortical receptive fields of alert monkeys. J Neurophysiol 43:444-468.

Gardner EP, Costanzo RM (1980c) Neuronal mechanisms underlying directional sensitivity of somatosensory cortical neurons in awake monkeys. J Neurophysiol 43:1342-1354.

Garraghty PE, Florence SL, Kaas JH (1990) Ablations of areas 3a and $3 \mathrm{~b}$ of monkey somatosensory cortex abolish cutaneous responsivity in area 1. Brain Res 528:165-169.

Hsiao SS, Johnson KO, O'Shaughnessy DM (1993) Effects of selective attention of spatial form processing in monkey primary and secondary somatosensory cortex. J Neurophysiol 70:444-447.

Hsiao SS, Johnson KO, Twombly IA, DiCarlo JJ (1996) Form processing and attention effects in somatosensory cortex. In: Somesthesis and the neurobiology of the somatosensory cortex (Franzen O, Johansson R, Terenius L, eds), pp 229-247. Basel: Birkhäuser Verlag.

Hubel DH, Wiesel TN (1962) Receptive fields, binocular interaction and functional architecture in the cat's visual cortex. J Physiol (Lond) 160:106-154.

Hubel DH, Wiesel TN (1968) Receptive fields and functional architecture of monkey striate cortex. J Physiol (Lond) 195:215-243.

Hyvarinen J, Poranen A (1978a) Movement-sensitive and direction and orientation-selective cutaneous receptive fields in the hand area of the post-central gyrus in monkeys. J Physiol (Lond) 283:523-537.

Hyvarinen J, Poranen A (1978b) Receptive field integration and submodality convergence in the hand area of the post-central gyrus of the alert monkey. J Physiol (Lond) 283:539-556.

Innocenti GM, Manzoni T (1972) Response patterns of somatosensory cortical neurones to peripheral stimuli. An intracellular study. Arch Ital Biol 110:322-347.

Iwamura Y, Inubushi S (1974) Regional diversity in excitatory and inhibitory receptive-field organization of cat thalamic ventrobasal neurons. J Neurophysiol 37:910-919.

Iwamura Y, Tanaka M, Sakamoto M, Hikosaka O (1983) Functional subdivisions representing different finger regions in area 3 of the first somatosensory cortex of the conscious monkey. Exp Brain Res $51: 315-326$.

Jänig W, Schoultz T, Spencer WA (1977) Temporal and spatial parameters of excitation and afferent inhibition in cuneothalamic relay neurons. J Neurophysiol 40:822-835.

Jänig W, Spencer WA, Younkin SG (1979) Spatial and temporal features of afferent inhibition of thalamocortical relays cells. J Neurophysiol 42:1450-1460.

Johansson RS, Westling G (1987) Signals in tactile afferents from the fingers eliciting adaptive motor responses during precision grip. Exp Brain Res 66:141-154.

Johnson KO (1980) Sensory discrimination: neural processes preceding discrimination decision. J Neurophysiol 43:1793-1815.

Johnson KO, Hsiao SS (1992) Tactile form and texture perception. Annu Rev Neurosci 15:227-250.

Johnson KO, Lamb GD (1981) Neural mechanisms of spatial tactile discrimination: neural patterns evoked by braille-like dot patterns in the monkey. J Physiol (Lond) 310:117-144.

Johnson KO, Phillips JR (1988) A rotating drum stimulator for scanning embossed patterns and textures across the skin. J Neurosci Methods 22:221-231.

Johnson KO, Hsiao SS, Twombly IA (1995) Neural mechanisms of tactile form recognition. In: The cognitive sciences (Gazzaniga M, ed), pp 253-268. Cambridge, MA: MIT.

Jones EG (1986) Connectivity of the primate sensory-motor cortex. In: Cerebral cortex, Vol 5 (Jones EG, Peters A, eds), pp 113-183. New York: Plenum.

Jones J, Palmer L (1987) The two-dimensional spatial structure of simple receptive fields in cat striate cortex. J Neurophysiol 58:1187-1211.

Kaas JH, Nelson RJ, Sur M, Merzenich MM (1984) Organization of somatosensory cortex in primates. In: Dynamic aspects of neocortical function (Edelman GM, Gall WE, Cowan WM, eds), pp 237-261. New York: Wiley.

LaMotte RH, Srinivasan MA (1987) Tactile discrimination of shape: responses of slowly adapting mechanoreceptive afferents to a step stroked across the monkey fingerpad. J Neurosci 7:1655-1671.

LaMotte RH, Whitehouse JM (1986) Tactile detection of a dot on a smooth surface: peripheral neural events. J Neurophysiol 56:1109-1128.

Laskin SE, Spencer WA (1979) Cutaneous masking. II. Geometry of excitatory and inhibitory receptive fields of single units in somatosensory cortex of the cat. J Neurophysiol 42:1061-1082.

Marmarelis PZ, Marmarelis VZ (1978) Analysis of physiological systems: the white noise approach. New York: Plenum.

Merzenich MM, Kaas JH, Sur M, Lin CS (1978) Double representation of the body surface within cytoarchitectonic areas $3 b$ and 1 in "SI" in the owl monkey (Aotus trivirgatus). J Comp Neurol 181:41-73.

Mountcastle VB (1984) Central nervous system mechanisms in mechanoreceptive sensibility. In: Handbook of physiology, Sec 1, Vol III, Pt 2 (Geiger SR, Darian-Smith I, Brookhart JM, Mountcastle VB, eds), pp 789-878. Baltimore: Waverly.

Mountcastle VB, Powell TP (1959) Neural mechanisms subserving cutaneous sensibility, with special reference to the role of afferent 
inhibition in sensory perception and discrimination. Bull Johns Hopkins Hosp 105:201-232.

Mountcastle VB, LaMotte RH, Carli G (1972) Detection thresholds for stimuli in humans and monkeys: comparison with threshold events in mechanoreceptive afferent nerve fibers innervating the monkey hand. J Neurophysiol 35:122-136.

Mountcastle VB, Reitboeck HJ, Poggio GF, Steinmetz MA (1991) Adaptation of the Reitboeck method of multiple microelectrode recording to the neocortex of the waking monkey. J Neurosci Methods 36:77-84.

Ochoa JL, Torebjörk HE (1983) Sensations evoked by intraneural microstimulation of single mechanoreceptor units innervating the human hand. J Physiol (Lond) 342:633-654.

Palmer LA, Jones JP, Stepnoski A (1991) Striate receptive fields as linear filters: characterization in two dimensions of space. In: Vision and visual dysfunction. The neural basis of visual function (Leventhal AG, ed), pp 246-265. Boca Raton, FL: CRC.

Paul RL, Merzenich MM, Goodman H (1972) Representation of slowly and rapidly adapting cutaneous mechanoreceptors of the hand in Brodmann's areas 3 and 1 of Macaca mulatta. Brain Res 36:229-249.

Phillips JR, Johnson KO (1981) Tactile spatial resolution: II. Neural representation of bars, edges, and gratings in monkey afferents. J Neurophysiol 46:1192-1203.

Phillips JR, Johnson KO, Hsiao SS (1988) Spatial pattern representation and transformation in monkey somatosensory cortex. Proc Natl Acad Sci USA 85:1317-1321.

Poggio GF, Mountcastle VB (1960) A study of the functional contributions of the lemniscal and spinothalamic systems to somatic sensibility. Bull Johns Hopkins Hosp 108:266-316.

Pons TP, Garraghty PE, Friedman DP, Mishkin M (1987) Physiological evidence for serial processing in somatosensory cortex. Science 237:417-420.

Pons TP, Garraghty PE, Mishkin M (1992) Serial and parallel processing of tactual information in somatosensory cortex of Rhesus monkeys. J Neurophysiol 68:518-527.

Powell TP, Mountcastle VB (1959a) The cytoarchitecture of the postcentral gyrus of the monkey Macaca mulatta. Bull Johns Hopkins Hosp 105:108-131.

Powell TP, Mountcastle VB (1959b) Some aspects of the functional organization of the cortex of the postcentral gyrus of the monkey: a correlation of findings obtained in a single unit analysis with cytoarchitecture. Bull Johns Hopkins Hosp 105:133-162.

Press WH, Teukolsky SA, Vetterling WT, Flannery BP (1992) Numerical recipes in FORTRAN: the art of scientific computing. Cambridge, U.K.: Cambridge UP.

Pubols LM, Leroy RF (1977) Orientation detectors in the primary somatosensory neocortex of the raccoon. Brain Res 129:61-74.

Randolph M, Semmes J (1974) Behavioral consequences of selective subtotal ablations in the postcentral gyrus of Macaca mulatta. Brain Res 70:55-70.

Recanzone GH, Merzenich MM, Jenkins WM, Grajski KA, Dinse HR (1992) Topographic reorganization of the hand representation in cortical area $3 \mathrm{~b}$ of owl monkeys trained in a frequency-discrimination task. J Neurophysiol 67:1031-1055.

Robinson CJ, Burton H (1980) Organization of somatosensory receptive fields in cortical areas $7 \mathrm{~b}$, retroinsula, postauditory and granular insula of M. fascicularis. J Comp Neurol 192:69-92.

Rockel AJ, Hiorns RW, Powell TP (1980) The basic uniformity in structure of the neocortex. Brain 103:221-244.

Shamma SA, Fleshman JW, Wiser PR, Versnel H (1993) Organization of response areas in ferret primary auditory cortex. J Neurophysiol 69:367-383.

Srinivasan MA, Whitehouse JM, LaMotte RH (1987) Detection of slip: peripheral neural coding. J Neurosci 7:1682-1697.

Sur M (1980) Receptive fields of neurons in areas 3b and 1 of somatosensory cortex in monkeys. Brain Res 198:465-471.

Sur M, Merzenich MM, Kaas JH (1980) Magnification, receptive-field area, and "hypercolumn" size in area $3 \mathrm{~b}$ and 1 of somatosensory cortex in owl monkeys. J Neurophysiol 44:295-311.

Sur M, Wall JT, Kaas JH (1984) Modular distribution of neurons with slowly adapting and rapidly adapting responses in area $3 \mathrm{~b}$ of somatosensory cortex in monkeys. J Neurophysiol 51:724-744.

Sur M, Garraghty PE, Bruce CJ (1985) Somatosensory cortex in macaque monkeys: laminar differences in receptive field size in areas $3 \mathrm{~b}$ and 1. Brain Res 342:391-395.

Sutter E (1987) A practical nonstochastic approach to nonlinear timedomain analysis. In: Advanced methods of physiological system modeling (Marmarelis VZ, ed). Los Angeles: USC.

Talbot WH, Darian-Smith I, Kornhuber HH, Mountcastle VB (1968) The sense of flutter-vibration: comparison of the human capacity with response patterns of mechanoreceptive afferents from the monkey hand. J Neurophysiol 31:301-334.

Vega-Bermudez F, Johnson KO, Hsiao SS (1991) Human tactile pattern recognition: active versus passive touch, velocity effects, patterns of confusion. J Neurophysiol 65:531-546.

Warren S, Hamalainen HA, Gardner EP (1986) Objective classification of motion- and direction-sensitive neurons in primary somatosensory cortex of awake monkeys. J Neurophysiol 56:598-622.

Whitehorn D, Towe AL (1968) Postsynaptic potential patterns evoked upon cells in sensorimotor cortex of cat by stimulation at the periphery. Exp Neurol 22:222-242.

Whitsel BL, Roppolo JR, Werner G (1972) Cortical information processing of stimulus motion on primate skin. J Neurophysiol 35:691717. 